\title{
Article \\ Exploitation of Tolerance of Wheat Kernel Weight and Shape-Related Traits from Aegilops tauschii under Heat and Combined Heat-Drought Stresses
}

\author{
Gamila Mohamed Idris Elhadi ${ }^{1}\left(\mathbb{D}\right.$, Nasrein Mohamed Kamal ${ }^{2,3}$, Yasir Serag Alnor Gorafi ${ }^{2,3}{ }^{(D)}$, Yuji Yamasaki ${ }^{2}$, \\ Kanenori Takata ${ }^{4,+}$, Izzat S. A. Tahir ${ }^{3}{ }^{(\mathbb{D}}$, Michel O. Itam ${ }^{1} \mathbb{(}$, , Hiroyuki Tanaka ${ }^{5}$ and Hisashi Tsujimoto ${ }^{2, *(D)}$ \\ 1 United Graduate School of Agricultural Sciences, Tottori University, Tottori 680-8553, Japan; \\ j_mohamed@live.com (G.M.I.E.); itammichaelo@gmail.com (M.O.I.) \\ 2 Arid Land Research Center, Tottori University, Tottori 680-0001, Japan; renokamal@tottori-u.ac.jp (N.M.K.); \\ yasirserag@tottori-u.ac.jp (Y.S.A.G.); yujyamas@tottori-u.ac.jp (Y.Y.) \\ 3 Wheat Research Program, Agricultural Research Corporation, P.O. Box 126, Wad Medani, Sudan; \\ izzatahir@yahoo.com \\ 4 National Agriculture and Food Research Organization, Fukuyama 721-8514, Japan; ktakata@obihiro.ac.jp \\ 5 Faculty of Agriculture, Tottori University, Tottori 680-8550, Japan; htanaka@tottori-u.ac.jp \\ * Correspondence: tsujim@tottori-u.ac.jp \\ + Present address: Obihiro University of Agriculture and Veterinary Medicine, Hokkaido 080-8555, Japan.
}

\section{check for} updates

Citation: Elhadi, G.M.I.; Kamal, N.M.; Gorafi, Y.S.A.; Yamasaki, Y.; Takata, K.; Tahir, I.S.A.; Itam, M.O.; Tanaka, H.; Tsujimoto, H. Exploitation of Tolerance of Wheat Kernel Weight and Shape-Related Traits from Aegilops tauschii under Heat and Combined Heat-Drought Stresses. Int. J. Mol. Sci. 2021, 22, 1830. https://doi.org/ $10.3390 /$ ijms 22041830

Academic Editor: Esther M. González

Received: 13 January 2021

Accepted: 9 February 2021

Published: 12 February 2021

Publisher's Note: MDPI stays neutral with regard to jurisdictional claims in published maps and institutional affiliations.

Copyright: (c) 2021 by the authors. Licensee MDPI, Basel, Switzerland. This article is an open access article distributed under the terms and conditions of the Creative Commons Attribution (CC BY) license (https:/ / creativecommons.org/licenses/by/ $4.0 /)$.
Abstract: Kernel weight and shape-related traits are inherited stably and increase wheat yield. Narrow genetic diversity limits the progress of wheat breeding. Here, we evaluated kernel weight and shape-related traits and applied genome-wide association analysis to a panel of wheat multiple synthetic derivative (MSD) lines. The MSD lines harbored genomic fragments from Aegilops tauschii. These materials were grown under optimum conditions in Japan, as well as under heat and combined heat-drought conditions in Sudan. We aimed to explore useful QTLs for kernel weight and shaperelated traits under stress conditions. These can be useful for enhancing yield under stress conditions. MSD lines possessed remarkable genetic variation for all traits under all conditions, and some lines showed better performance than the background parent Norin 61. We identified 82 marker trait associations (MTAs) under the three conditions; most of them originated from the D genome. All of the favorable alleles originated from Ae. tauschii. For the first time, we identified markers on chromosome 5D associated with a candidate gene encoding a RING-type E3 ubiquitin-protein ligase and expected to have a role in regulating wheat seed size. Our study provides important knowledge for the improvement of wheat yield under optimum and stress conditions. The results emphasize the importance of Ae. tauschii as a gene reservoir for wheat breeding.

Keywords: Aegilops tauschii; heat stress; combined heat-drought stress; kernel weight; GWAS; grain yield

\section{Introduction}

Abiotic stresses such as heat and drought affect yield negatively by reducing grain size and number. While the stresses in any growth stage affect the final yield, those at the grain filling stage are the most crucial [1,2]. Heat and drought stress in all developmental stages influence important morphological traits [3,4]. To adapt to these harsh environments, great efforts have been made to produce genetically tolerant plants and to understand the mechanisms behind the stress tolerance [5,6]. To identify genomic regions responsible for grain yield under stress conditions, analysis of the yield components is crucial [7]. Kernel weight is considered the most important and heritable trait among these components [8]. This trait is closely associated with kernel shape-related traits, such as kernel length, kernel width and kernel diameter [9]. The improvement of kernel weight and shape-related traits under stressed conditions is a promising approach to increasing wheat production [10]. In 
addition, screening for shape-related traits using image analysis can provide an easy and accurate means to assess yield components.

Wheat genetic diversity has been narrowed down in extensive breeding programs and thus, finding new genetic diversity in wild species is indispensable for improvement [11,12]. Many studies have used Aegilops species as genetic resources and reported their tolerance to abiotic stresses $[13,14]$. Among the related wild species, Aegilops tauschii is the most promising species because it has a $\mathrm{D}$ genome common with that of bread wheat and because no special cytological technique is needed to induce homologous recombination [15]. In this study, we employed wheat multiple synthetic derivative (MSD) lines, which contain the genetic diversity of a large accession of a wild species, Aegilops tauschii, and are suitable materials for genetic analysis [16].

Great progress has been made in identifying major QTLs for kernel size and shape [17-22], and several candidate genes were identified in wheat. For instance, cytokinin oxidase (encoded by $T a C K X)$ reversibly inactivates cytokinin and increases kernel weight [23]. Cell wall invertase (encoded by TaGW1) regulates kernel size by sink tissue development [24], and RING-type E3 ubiquitin-protein ligase (encoded by $\mathrm{TaGW}$ ) increases kernel weight and size [25].

These studies describe kernel development under normal conditions, but extensive studies under stress conditions have not been conducted. To breed wheat genotypes that maintain grain yield even under stress conditions, knowledge of genotypic and environment interaction is necessary. Therefore, this study aimed at identifying the phenotypic and genotypic variation of kernel weight and shape-related traits among MSD lines under optimum (OP), heat $(\mathrm{H})$ and combined heat-drought $(\mathrm{HD})$ conditions, and to reveal the genetic mechanism of the productivity under stress, from the view point of kernel traits. Our results revealed a great diversity among the MSD lines in kernel weight and other kernel shape-related traits under all conditions. We identified promising markers and alleles that will contribute to our understanding of productivity under stressed condition and could be used in wheat breeding after validation.

\section{Results}

2.1. Phenotypic Variation of Kernel Weight and Shape-Related Traits under Optimum, Heat, and Combined Heat-Drought Conditions

MSD lines showed large phenotypic variation of all kernel traits under all three optimum $(\mathrm{OP})$, heat $(\mathrm{H})$ and combined heat-drought $(\mathrm{HD})$ conditions (Figure 1). The MSD lines showed a wider range of variation under $\mathrm{H}$ and $\mathrm{HD}$ than under OP conditions, reflecting the MSD response to the stress conditions (Figure 1). The values of the kernel weight and shape traits were reduced under $\mathrm{H}$ and $\mathrm{HD}$ conditions, resulting in the means shifting towards the low values. The effects on kernel weight, kernel diameter, kernel size and kernel width were more potent than those on kernel length and kernel circularity (Figure 1).

Under OP conditions, the genotypic effect was significant for all traits except kernel width (Table 1). Differences were significant between the two seasons (S) in all traits except kernel length, and the interaction $(G \times S)$ was significant for all traits (Table 1). Under $\mathrm{H}$ and $\mathrm{HD}$ conditions, differences among genotypes were significant for all traits. The environment (E) affected all traits except kernel length, and $G \times E$ had no significant differences in any traits (Table 1$)$. Moderate heritability $\left(H^{2}\right)(0.42$ to 0.67$)$ was observed under OP conditions for all traits except kernel width, which had a low heritability value (0.29). In contrast, high $H^{2}$ was observed for all the traits under the stress environments (0.88 to 0.97 ) (Table 1$)$. 

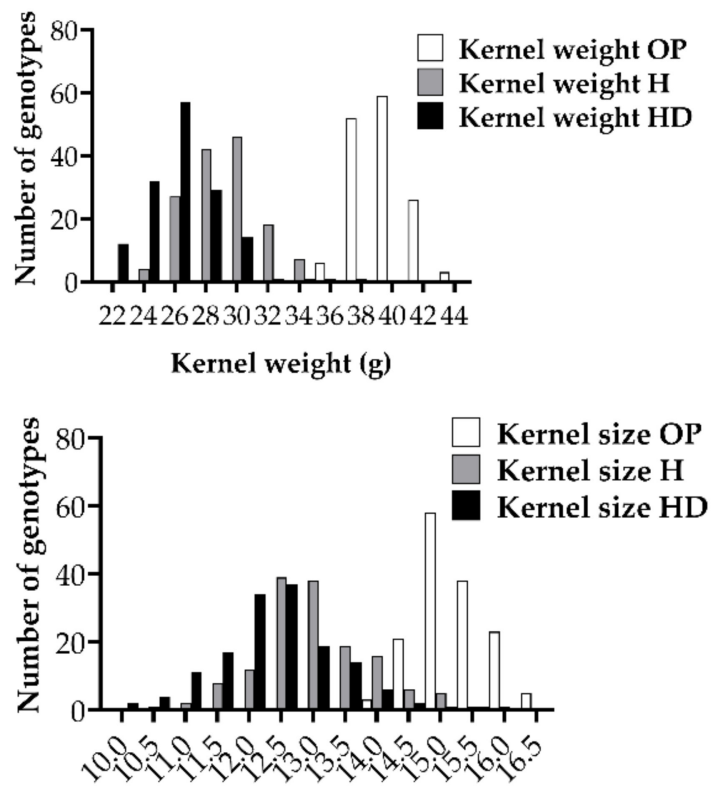

Kernel size $\left(\mathrm{mm}^{2}\right)$
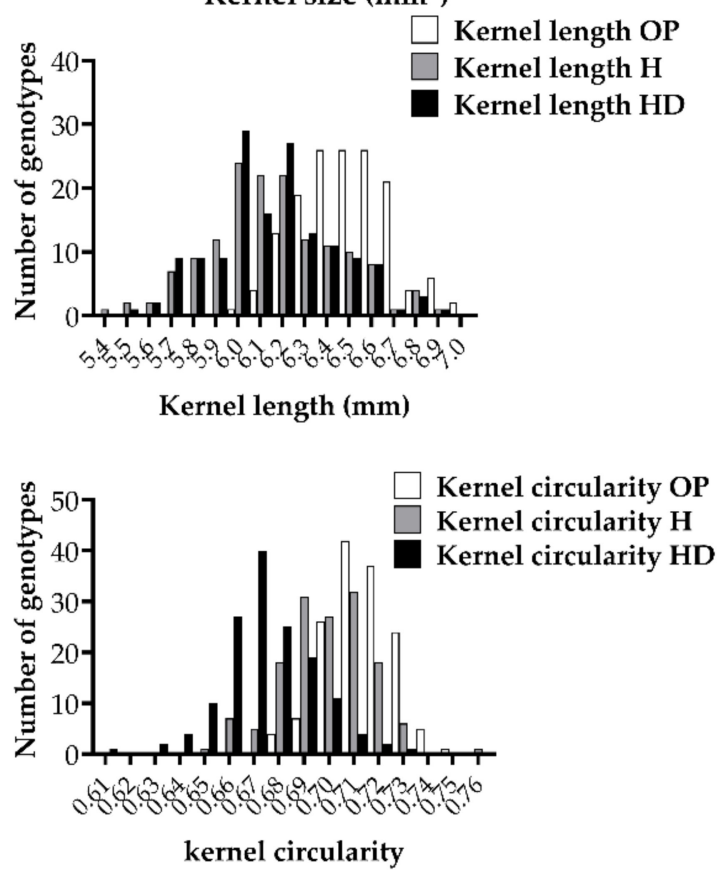

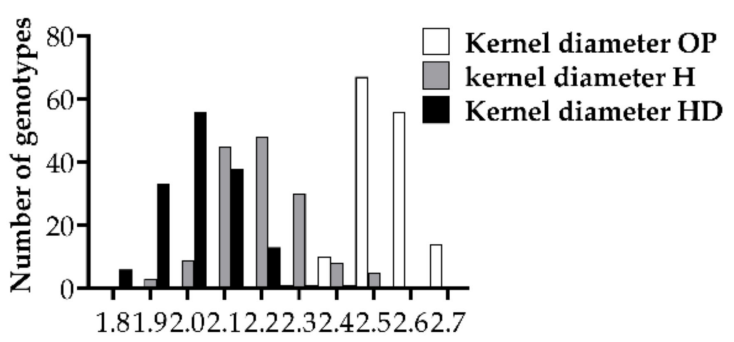

Kernel diameter (mm)

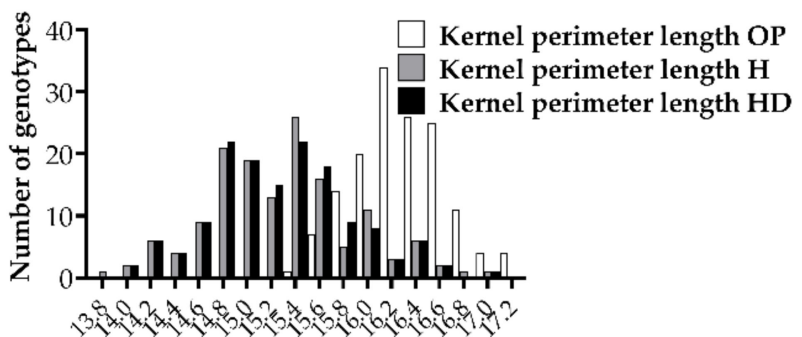

Kernel perimeter length (mm)

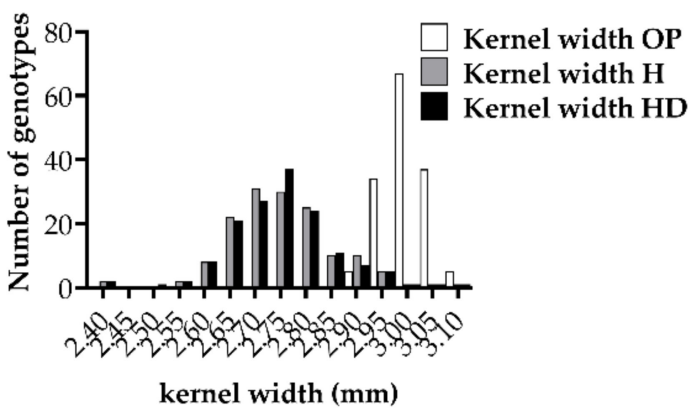

Figure 1. Frequency distribution of the multiple synthetic derivative lines grown under optimum (OP), heat (H) and combined heat-drought (HD) conditions. For the OP conditions, the predicted means of the values from two seasons, S1 and S2, were used. 
Table 1. Analysis of variance and heritability of kernel weight and shape-related traits under optimum (OP), heat (H) and combined heat-drought (HD) conditions for multiple synthetic derivatives lines.

\begin{tabular}{|c|c|c|c|c|c|c|c|c|c|}
\hline & \multicolumn{3}{|c|}{ Kernel Weight (g) } & \multicolumn{3}{|c|}{ Kernel Diameter (mm) } & \multicolumn{3}{|c|}{ Kernel Size $\left(\mathrm{mm}^{2}\right)$} \\
\hline & $\mathrm{SED} \pm$ & $H^{2}$ & $p$-value & $\mathrm{SED} \pm$ & $H^{2}$ & $p$-value & $\mathrm{SED} \pm$ & $H^{2}$ & $p$-value \\
\hline $\mathrm{C}(\mathrm{OP})$ & 3.33 & - & $* * *$ & 0.13 & - & $* * *$ & 1.94 & & ** \\
\hline $\begin{array}{l}\text { S }(\mathrm{S} 1 \times \\
\text { S2) }\end{array}$ & - & - & $*$ & - & - & $* * *$ & - & - & $* *$ \\
\hline $\mathrm{G} \times \mathrm{S}$ & 3.39 & 0.56 & $* * *$ & 0.13 & 0.64 & $* * *$ & 1.94 & 0.42 & $* *$ \\
\hline $\mathrm{H}$ & 1.53 & - & $* * *$ & 0.08 & - & $* * *$ & 0.60 & - & $* * *$ \\
\hline HD & 1.73 & - & $* * *$ & 0.09 & - & $* * *$ & 0.61 & - & $* * *$ \\
\hline $\mathrm{E}$ & - & - & $* * *$ & - & - & $* * *$ & - & - & $* * *$ \\
\hline \multirow[t]{3}{*}{$\mathrm{G} \times \mathrm{E}$} & 1.75 & 0.92 & ns & 0.07 & 0.88 & ns & 0.55 & 0.94 & ns \\
\hline & \multicolumn{3}{|c|}{ Kernel Length (mm) } & \multicolumn{3}{|c|}{ Kernel Width (mm) } & \multicolumn{3}{|c|}{ Kernel Circularity } \\
\hline & $\mathrm{SED} \pm$ & $H^{2}$ & $p$-value & $\mathrm{SED} \pm$ & $H^{2}$ & $p$-value & $\mathrm{SED} \pm$ & $H^{2}$ & $p$-value \\
\hline $\mathrm{C}(\mathrm{OP})$ & 0.46 & - & $* * *$ & 0.22 & & ns & 0.02 & - & $* * *$ \\
\hline $\begin{array}{c}\mathrm{S}(\mathrm{S} 1 \times \\
\mathrm{S} 2)\end{array}$ & - & - & ns & - & - & $* * *$ & - & - & $* *$ \\
\hline $\mathrm{G} \times \mathrm{S}$ & 0.46 & 0.51 & $* * *$ & 0.22 & 0.29 & $*$ & 0.02 & 0.67 & $* *$ \\
\hline $\mathrm{H}$ & 0.13 & - & $* * *$ & 0.07 & - & $* * *$ & 0.01 & - & $* * *$ \\
\hline HD & 0.18 & - & $* * *$ & 0.08 & - & $* * *$ & 0.01 & - & $* * *$ \\
\hline $\mathrm{E}$ & - & - & ns & - & - & $* * *$ & - & - & $* * *$ \\
\hline $\mathrm{G} \times \mathrm{E}$ & 0.14 & 0.97 & ns & 0.07 & 0.88 & ns & 0.01 & 0.92 & ns \\
\hline
\end{tabular}

C: combined analysis for the two seasons under OP conditions; SED \pm : standard error of differences; ns, not significant; ${ }^{*} p<0.05,{ }^{* *} p<0.01$, $* * * p<0.001$.

All kernel traits except kernel circularity correlated positively with kernel weight under all conditions; the correlation with kernel diameter was the strongest (Figure 2). Correlations were positive among kernel size, kernel perimeter length and kernel length (Figure 2).

To understand the performance of MSD lines under stress conditions (H and HD), we calculated the heat susceptibility index (HSI) and the combined heat-drought susceptibility index (HDSI) on the basis of the traits most strongly correlated with kernel weight, i.e., kernel diameter and kernel size. We performed a regression analysis between the HSI and HDSI to identify any relationship between the performances of MSD lines under the different stresses (Figure 3). There was an association between the MSD lines' performance under $\mathrm{H}$ and HD conditions $\left(R^{2}=0.45,0.33\right.$ and 0.53 for kernel weight, kernel diameter and kernel size, respectively). The HSI and HDSI of the background parent of the MSD lines Norin 61 and leading Sudanese cultivar Imam were around 1 (Figure 3), indicating that these genotypes showed levels of reduction similar to all genotypes studied, as seen in Figure 1.

Interestingly, some MSD lines (indicated with red color) showed stable performance under stress conditions; they had the highest kernel weight, kernel diameter and kernel size under $\mathrm{H}$ and HD stress conditions (Figure 3). MSD187 performed well under all conditions in all three traits, whereas MSD259 was much more strongly affected by H and HD stresses than Norin 6" and Imam (Figures 3 and 4). 
(a)

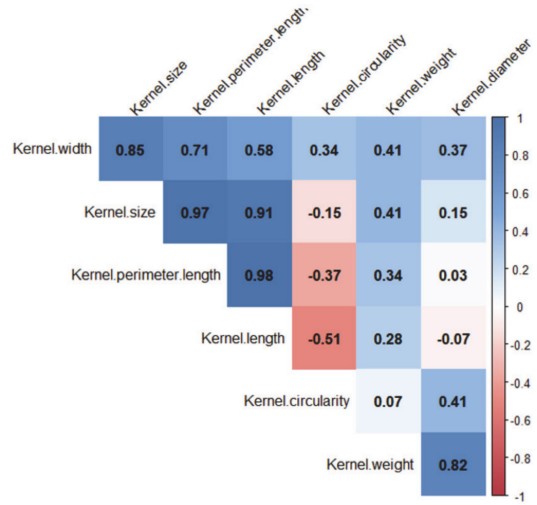

(c)

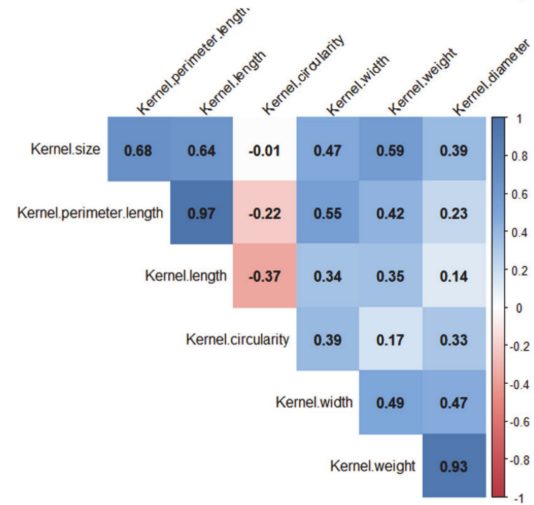

(b)

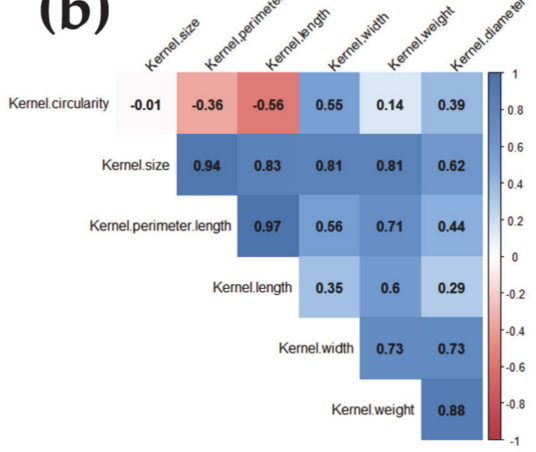

Figure 2. Correlation coefficient for kernel weight and shape-related traits under (a) optimum; (b) heat; and (c) combined heat-drought conditions. Absolute values $>0.15$ were significant at $p=0.05 ;>0.20$ were significant at $p=0.01$ under optimum, heat and combined heat-drought conditions, whereas absolute values $>26,29$ and 27 were significant at $p=0.001$ under optimum, heat and combined heat-drought conditions, respectively.

(a)

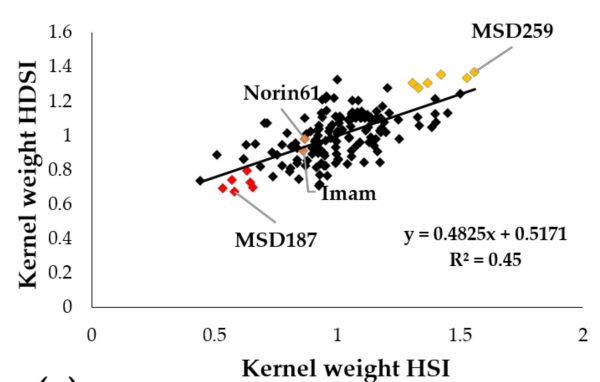

(c)

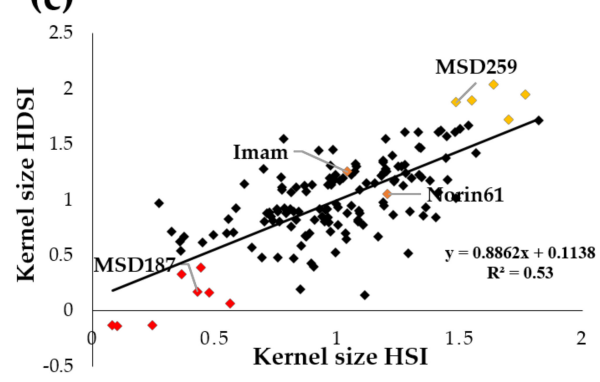

(b)

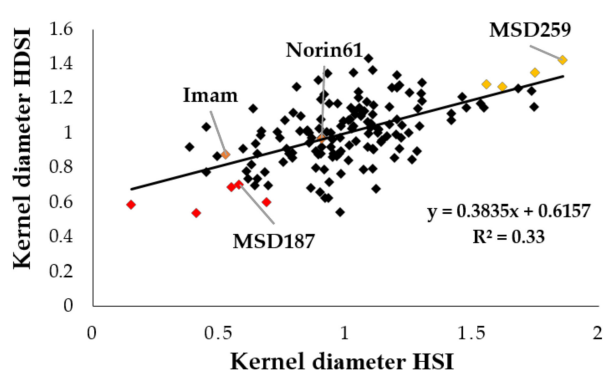

Figure 3. Regression analysis of the relationship between the heat susceptibility index (HSI) and the combined heat-drought susceptibility index (HDSI) for kernel (a) weight; (b) diameter; and (c) area size. Red color indicates tolerant lines with an index value of about 0.5 and yellow color indicates sensitive lines with an index value higher than one. The genetic background parent of the MSD lines Norin 61, the leading Sudanese cultivar Imam and two representative MSD lines showing high (MSD259) and low (MSD187) reductions are indicated. 


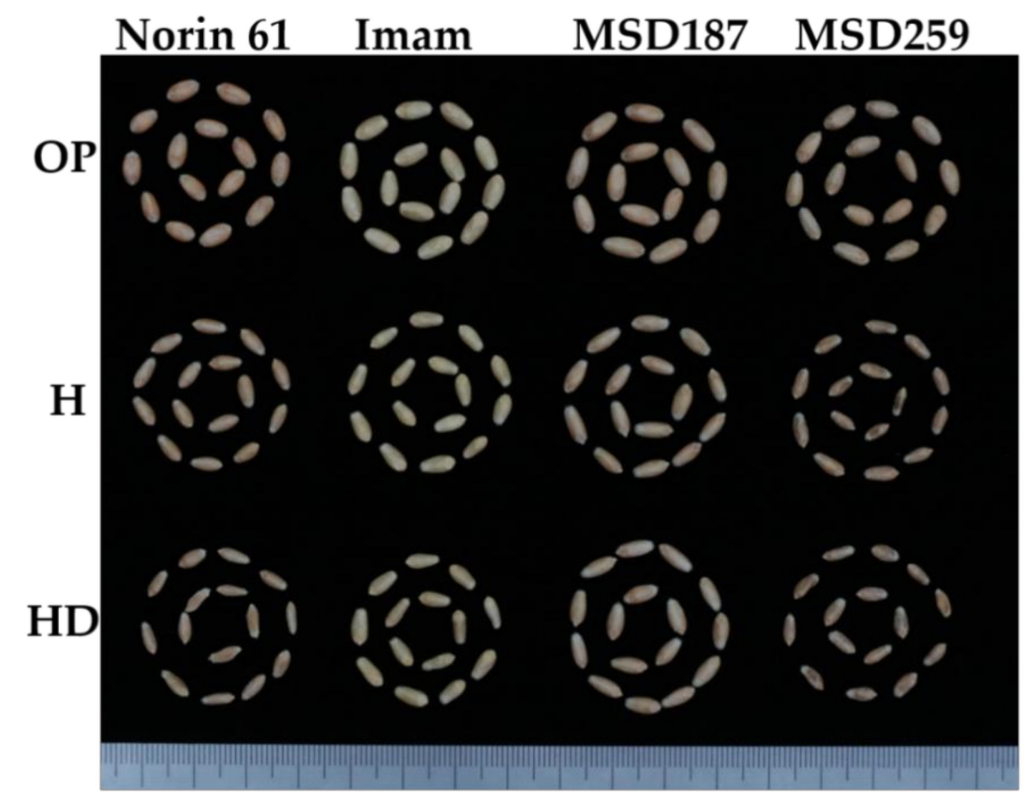

Figure 4. Variations in kernel weight and shape-related traits under optimum (OP), heat $(\mathrm{H})$ and combined heat-drought (HD) conditions. Norin 61: the background of the MSD lines, showed a reduction under $\mathrm{H}$ and $\mathrm{HD}$ conditions; Imam: check cultivar known as heat tolerant, showed a small reduction; MSD187: showed a slight reduction under H and HD conditions; MSD259: highly sensitive line, showed remarkable reduction under $\mathrm{H}$ and HD conditions.

\subsection{Marker Trait Associations for Kernel Weight and Shape-Related Traits}

We calculated the best linear unbiased predictions (BLUPs) for OP conditions with the data from two seasons, considering the significant seasonal effect in the association analysis. We identified 82 marker trait associations (MTAs) on different chromosomes under all conditions and for HSI and HDSI (Figures S1 and S2). Among the A, B and D genomes, the D genome had the highest MTA number under all conditions (Figure S3a).

Under OP conditions, the A, B and D genomes contributed $30 \%, 13 \%$ and $57 \%$, respectively (Figure S3b). Significant markers associated with kernel weight and kernel diameter were found on chromosome 6A and those associated with kernel weight, kernel size, kernel length and kernel perimeter length were found on chromosome 5D (Figure S4, Table 2). Significant markers on chromosome 3B were associated with kernel length, kernel perimeter length and kernel circularity, while those on 7B and 7D were associated with kernel width and those on 2B and 7A with kernel length (Figure S4, Table 2). Chromosome 2D had the highest number of MTAs, most of them associated with kernel circularity. The contribution $\left(R^{2}\right)$ of markers ranged from 0.074 in marker 1113863, associated with kernel diameter, on chromosome 2A to 0.16 in marker 1088563, associated with kernel circularity, on chromosome 2D (Table 2). Marker 5357358, on chromosome 2A, affected kernel size and kernel perimeter length (Figure 4, Table 2). In addition, markers 3028836 and 1088488 | F I 027 , on chromosomes $5 \mathrm{~A}$ and $5 \mathrm{D}$, respectively, affected kernel size, kernel length and kernel perimeter length (Figure 5a, Table 2). Marker 4395641, on chromosome 6A, affected kernel diameter and kernel weight (Figure 5a, Table 2). In total, 30 MTAs were obtained under OP conditions (Table 2); some of them had pleiotropic effects, some were common between OP and $\mathrm{HD}$ conditions and others were associated with OP conditions (Table 2). 
Table 2. Marker trait associations of kernel weight and shape-related traits in multiple synthetic derivative lines grown under optimum (OP), heat (H) and combined heat-drought (HD) conditions.

\begin{tabular}{|c|c|c|c|c|c|c|}
\hline Chromosome & $\begin{array}{c}\text { Marker } \\
\text { Position (Bp) }\end{array}$ & Marker & Trait & Conditions & $p$-Value & $R^{2}$ \\
\hline $1 \mathrm{~A}$ & 229673410 & 1115856 | F | 0-14 & Kernel circularity & $\mathrm{H}$ & $9.75 \times 10^{-4}$ & 0.10 \\
\hline \multirow{8}{*}{$2 \mathrm{~A}$} & 1696501 & $3026123|\mathrm{~F}| 0-8$ & Kernel weight & HD & $2.40 \times 10^{-4}$ & 0.10 \\
\hline & \multirow{2}{*}{17036238} & \multirow{2}{*}{5357358} & Kernel size & OP & $2.49 \times 10^{-4}$ & 0.12 \\
\hline & & & Kernel perimeter length & $\mathrm{OP}$ & $7.51 \times 10^{-4}$ & 0.10 \\
\hline & 57562452 & 3938604 & Kernel width & HD & $1.08 \times 10^{-3}$ & 0.09 \\
\hline & 149553891 & 1113863 & Kernel diameter & $\mathrm{OP}$ & $1.67 \times 10^{-3}$ & 0.07 \\
\hline & \multirow{2}{*}{250645685} & \multirow{2}{*}{1110845} & Kernel diameter & HD & $9.00 \times 10^{-4}$ & 0.08 \\
\hline & & & Kernel weight & HD & $7.72 \times 10^{-4}$ & 0.08 \\
\hline & 244243147 & 5010926 & Kernel size & HD & $1.02 \times 10^{-3}$ & 0.08 \\
\hline $3 \mathrm{~A}$ & 39514910 & 1035262 | F | 0-46 & Kernel circularity & HD & $8.80 \times 10^{-5}$ & 0.11 \\
\hline \multirow[b]{2}{*}{$4 \mathrm{~A}$} & \multirow{2}{*}{184463076} & \multirow{2}{*}{987701} & \multirow{2}{*}{ Kernel circularity } & $\mathrm{H}$ & $1.01 \times 10^{-3}$ & 0.08 \\
\hline & & & & HD & $8.28 \times 10^{-4}$ & 0.09 \\
\hline \multirow{8}{*}{$5 \mathrm{~A}$} & \multirow{2}{*}{117490096} & \multirow{2}{*}{1079158} & kernel length & OP & $5.02 \times 10^{-4}$ & 0.10 \\
\hline & & & Kernel perimeter length & OP & $5.47 \times 10^{-4}$ & 0.10 \\
\hline & \multirow{3}{*}{$\begin{array}{l}117719229 \\
118132341\end{array}$} & 1157204 | F | 0-51 & Kernel perimeter length & OP & $9.12 \times 10^{-4}$ & 0.10 \\
\hline & & \multirow[t]{2}{*}{$977527|\mathrm{~F}| 0-25$} & Kernel perimeter length & OP & $9.13 \times 10^{-4}$ & 0.10 \\
\hline & & & Kernel size & OP & $5.09 \times 10^{-4}$ & 0.10 \\
\hline & \multirow[t]{2}{*}{124441377} & \multirow[t]{2}{*}{3028836} & Kernel length & $\mathrm{OP}$ & $6.30 \times 10^{-4}$ & 0.09 \\
\hline & & & Kernel perimeter length & OP & $3.78 \times 10^{-4}$ & 0.10 \\
\hline & 127107886 & 3940004 | F | 0-21 & Kernel width & HD & $1.11 \times 10^{-4}$ & 0.19 \\
\hline \multirow{12}{*}{$6 \mathrm{~A}$} & 945226 & 2277231 & Kernel circularity & $\mathrm{H}$ & $7.23 \times 10^{-4}$ & 0.09 \\
\hline & \multirow{2}{*}{10617494} & \multirow{2}{*}{4395641} & Kernel weight & OP & $2.94 \times 10^{-4}$ & 0.10 \\
\hline & & & Kernel diameter & OP & $1.67 \times 10^{-3}$ & 0.08 \\
\hline & 12184855 & 1115036 & Kernel weight & OP & $5.48 \times 10^{-4}$ & 0.09 \\
\hline & 15813131 & 4407451 & Kernel weight & $\mathrm{OP}$ & $1.99 \times 10^{-4}$ & 0.10 \\
\hline & 32701094 & $1019857|\mathrm{~F}| 0-23$ & Kernel diameter & $\mathrm{H}$ & $2.03 \times 10^{-4}$ & 0.10 \\
\hline & 77372430 & 7327852 & Kernel size & HD & $4.35 \times 10^{-4}$ & 0.09 \\
\hline & 86323044 & 3945281 & Kernel diameter & $\mathrm{H}$ & $4.39 \times 10^{-4}$ & 0.09 \\
\hline & \multirow[t]{2}{*}{133562282} & \multirow[t]{2}{*}{3384829} & Kernel size & HD & $6.62 \times 10^{-4}$ & 0.09 \\
\hline & & & Kernel weight & $\mathrm{H}$ & $7.75 \times 10^{-4}$ & 0.09 \\
\hline & \multirow[t]{2}{*}{145087238} & 5968258 & Kernel diameter & $\mathrm{H}$ & $5.89 \times 10^{-4}$ & 0.10 \\
\hline & & & Kernel perimeter length & HD & $9.15 \times 10^{-4}$ & 0.08 \\
\hline $7 \mathrm{~A}$ & 160041813 & 1056001 & Kernel length & $\mathrm{OP}$ & $9.34 \times 10^{-4}$ & 0.10 \\
\hline $2 \mathrm{~B}$ & 118229752 & $7351021|\mathrm{~F}| 0-10$ & Kernel length & $\mathrm{OP}$ & $5.20 \times 10^{-4}$ & 0.09 \\
\hline & & & Kernel length & $\mathrm{OP}$ & $1.21 \times 10^{-4}$ & 0.12 \\
\hline & 24450611 & 3958195 & Kernel perimeter length & OP & $3.86 \times 10^{-4}$ & 0.10 \\
\hline 3B & 678386865 & 4396161 & Kernel circularity & OP & $9.64 \times 10^{-4}$ & 0.08 \\
\hline & 755958103 & 7353565 & Kernel size & HD & $8.84 \times 10^{-4}$ & 0.09 \\
\hline & 109565358 & 1302570 & Kernel circularity & HD & $4.34 \times 10^{-4}$ & 0.11 \\
\hline $5 B$ & & & Kernel weight & HD & $5.07 \times 10^{-4}$ & 0.11 \\
\hline & 196141955 & $2248796|\mathrm{~F}| 0-42$ & Kernel size & HD & $2.91 \times 10^{-4}$ & 0.12 \\
\hline & 48765954 & 3020427 & Kernel size & HD & $5.31 \times 10^{-4}$ & 0.11 \\
\hline $6 \mathrm{~B}$ & 191946279 & 993061 & Kernel circularity & HD & $6.60 \times 10^{-4}$ & 0.10 \\
\hline & 205492257 & 5581272 & Kernel size & HD & $1.80 \times 10^{-4}$ & 0.11 \\
\hline $7 \mathrm{~B}$ & 240968931 & 3949081 & Kernel width & OP & $6.80 \times 10^{-4}$ & 0.10 \\
\hline & 246168466 & 4260892 & Kernel width & OP & $7.13 \times 10^{-4}$ & 0.10 \\
\hline
\end{tabular}


Table 2. Cont

\begin{tabular}{|c|c|c|c|c|c|c|}
\hline Chromosome & $\begin{array}{c}\text { Marker } \\
\text { Position (Bp) }\end{array}$ & Marker & Trait & Conditions & $p$-Value & $R^{2}$ \\
\hline \multirow{16}{*}{$2 \mathrm{D}$} & 9318714 & 4999702 & Kernel width & OP & $9.34 \times 10^{-4}$ & 0.08 \\
\hline & \multirow{3}{*}{10253062} & \multirow{3}{*}{1000563 | F | 0-24 } & Kernel length & OP & $1.00 \times 10^{-4}$ & 0.14 \\
\hline & & & Kernel perimeter length & OP & $3.28 \times 10^{-4}$ & 0.12 \\
\hline & & & Kernel circularity & OP & $2.54 \times 10^{-4}$ & 0.14 \\
\hline & \multirow[t]{2}{*}{142248333} & \multirow[t]{2}{*}{3946155} & Kernel circularity & HD & $2.48 \times 10^{-4}$ & 0.11 \\
\hline & & & Kernel width & $\mathrm{OP}$ & $2.20 \times 10^{-4}$ & 0.11 \\
\hline & \multirow[b]{2}{*}{142982233} & \multirow[b]{2}{*}{3957018} & Kernel circularity & OP & $7.92 \times 10^{-5}$ & 0.14 \\
\hline & & & Kernel size & HD & $7.55 \times 10^{-4}$ & 0.09 \\
\hline & \multirow{2}{*}{143867561} & \multirow{2}{*}{4542702} & Kernel circularity & $\mathrm{OP}$ & $1.25 \times 10^{-4}$ & 0.14 \\
\hline & & & Kernel circularity & OP & $3.95 \times 10^{-4}$ & 0.14 \\
\hline & 10453150 & 1076033 | F | 0-62 & Kernel width & HD & 0.00107 & 0.08 \\
\hline & 143981582 & 3534099 & Kernel width & $\mathrm{OP}$ & $6.51 \times 10^{-4}$ & 0.09 \\
\hline & 147520289 & 1088563 & Kernel circularity & $\mathrm{OP}$ & $3.21 \times 10^{-5}$ & 0.16 \\
\hline & 147746144 & 4992154 | F | 0-9 & Kernel circularity & OP & $3.14 \times 10^{-4}$ & 0.15 \\
\hline & 149031034 & $1116168|\mathrm{~F}| 0-15$ & Kernel circularity & OP & $2.72 \times 10^{-4}$ & 0.13 \\
\hline & 149318699 & 3023828 & Kernel circularity & $\mathrm{OP}$ & $4.58 \times 10^{-4}$ & 0.12 \\
\hline \multirow{3}{*}{$3 \mathrm{D}$} & 762269 & 3385313 |F | 0-6 & Kernel length & HD & $8.41 \times 10^{-4}$ & 0.10 \\
\hline & 112899800 & $1203228|\mathrm{~F}| 0-45$ & Kernel circularity & $\mathrm{H}$ & $1.01 \times 10^{-3}$ & 0.11 \\
\hline & 113047617 & $1122898|\mathrm{~F}| 0-41$ & Kernel circularity & $\mathrm{H}$ & $4.03 \times 10^{-4}$ & 0.13 \\
\hline \multirow{2}{*}{$4 \mathrm{D}$} & \multirow[b]{2}{*}{27756435} & \multirow{2}{*}{1001438 | F | 0-46 } & Kernel length & $\mathrm{H}$ & $1.09 \times 10^{-3}$ & 0.10 \\
\hline & & & Kernel length & HD & $8.93 \times 10^{-4}$ & 0.10 \\
\hline \multirow{28}{*}{$5 \mathrm{D}$} & 59337192 & $1099271|\mathrm{~F}| 0-23$ & Kernel weight & HD & $9.63 \times 10^{-4}$ & 0.10 \\
\hline & 112083542 & 1091823 | F | 0-41 & Kernel weight & $\mathrm{H}$ & $9.80 \times 10^{-4}$ & 0.10 \\
\hline & 117708268 & 1072444 & Kernel size & HD & $8.30 \times 10^{-4}$ & 0.09 \\
\hline & & & Kernel size & $\mathrm{H}$ & $6.65 \times 10^{-4}$ & 0.09 \\
\hline & 118171820 & 2244825 & Kernel size & HD & $1.09 \times 10^{-3}$ & 0.09 \\
\hline & & & Kernel perimeter length & HD & $5.62 \times 10^{-4}$ & 0.09 \\
\hline & \multirow{4}{*}{119535444} & \multirow{4}{*}{991465} & Kernel weight & HD & $4.95 \times 10^{-4}$ & 0.09 \\
\hline & & & Kernel size & HD & $1.39 \times 10^{-5}$ & 0.15 \\
\hline & & & Kernel length & HD & $7.49 \times 10^{-4}$ & 0.09 \\
\hline & & & Kernel perimeter length & HD & $7.08 \times 10^{-4}$ & 0.09 \\
\hline & 120017850 & $1201315|\mathrm{~F}| 0-67$ & Kernel diameter & HD & $4.98 \times 10^{-4}$ & 0.13 \\
\hline & 120914450 & $7351647|\mathrm{~F}| 0-44$ & Kernel size & HD & $4.90 \times 10^{-4}$ & 0.11 \\
\hline & \multirow[t]{2}{*}{121111673} & \multirow[t]{2}{*}{4910927} & Kernel weight & $\mathrm{OP}$ & $1.05 \times 10^{-3}$ & 0.08 \\
\hline & & & Kernel size & OP & $2.82 \times 10^{-4}$ & 0.13 \\
\hline & \multirow[t]{2}{*}{138730779} & \multirow[t]{2}{*}{1088488 | F | 0-27 } & Kernel length & OP & $3.89 \times 10^{-4}$ & 0.12 \\
\hline & & & Kernel perimeter length & OP & $1.69 \times 10^{-4}$ & 0.13 \\
\hline & \multirow{2}{*}{139543900} & & Kernel weight & $\mathrm{H}$ & $8.05 \times 10^{-4}$ & 0.09 \\
\hline & & 5332404 & Kernel size & HD & $2.38 \times 10^{-4}$ & 0.11 \\
\hline & 140397428 & $3955838|\mathrm{~F}| 0-28$ & Kernel size & HD & $6.41 \times 10^{-4}$ & 0.12 \\
\hline & 140551286 & 1101952 & Kernel size & HD & $2.07 \times 10^{-4}$ & 0.12 \\
\hline & 140719410 & 7350532 & Kernel size & HD & $5.78 \times 10^{-4}$ & 0.09 \\
\hline & 141061760 & 1087740 & Kernel size & HD & $2.32 \times 10^{-4}$ & 0.11 \\
\hline & 141600686 & 1215969 & Kernel size & HD & $6.05 \times 10^{-5}$ & 0.12 \\
\hline & 142135700 & 3941995 & Kernel size & HD & $4.32 \times 10^{-4}$ & 0.13 \\
\hline & 142429952 & 3946915 & Kernel size & HD & $1.08 \times 10^{-3}$ & 0.09 \\
\hline & 142680984 & 3954584 & Kernel size & HD & $6.87 \times 10^{-5}$ & 0.13 \\
\hline & 143115886 & 3026564 & Kernel size & HD & $7.86 \times 10^{-5}$ & 0.13 \\
\hline & 143728332 & 6041628 & Kernel size & HD & $6.01 \times 10^{-5}$ & 0.12 \\
\hline
\end{tabular}


Table 2. Cont

\begin{tabular}{|c|c|c|c|c|c|c|}
\hline Chromosome & $\begin{array}{c}\text { Marker } \\
\text { Position (Bp) }\end{array}$ & Marker & Trait & Conditions & $p$-Value & $R^{2}$ \\
\hline & \multirow{2}{*}{144829140} & \multirow[b]{2}{*}{$1398977|\mathrm{~F}| 0-23$} & Kernel weight & $\mathrm{H}$ & $6.45 \times 10^{-4}$ & 0.11 \\
\hline & & & Kernel size & HD & $6.21 \times 10^{-5}$ & 0.17 \\
\hline & 145561848 & \multirow{2}{*}{6044286} & Kernel weight & $\mathrm{H}$ & $3.88 \times 10^{-4}$ & 0.11 \\
\hline & 145561848 & & Kernel size & HD & $1.64 \times 10^{-5}$ & 0.17 \\
\hline & 145561848 & 1696241 & Kernel size & HD & $5.62 \times 10^{-4}$ & 0.09 \\
\hline & \multirow[t]{3}{*}{148482930} & \multirow[t]{2}{*}{2257612 | F | 0-47 } & Kernel size & HD & $4.10 \times 10^{-4}$ & 0.09 \\
\hline & & & Kernel size & $\mathrm{OP}$ & $8.53 \times 10^{-4}$ & 0.08 \\
\hline & & \multirow{4}{*}{ 1073897 | F | 0-27 } & Kernel size & $\mathrm{H}$ & $8.24 \times 10^{-4}$ & 0.08 \\
\hline & \multirow[t]{3}{*}{150113559} & & Kernel size & HD & $2.54 \times 10^{-4}$ & 0.09 \\
\hline & & & Kernel length & HD & $5.84 \times 10^{-4}$ & 0.08 \\
\hline & & & Kernel perimeter length & HD & $3.56 \times 10^{-4}$ & 0.09 \\
\hline & 151218934 & $1218899|\mathrm{~F}| 0-6$ & Kernel size & HD & $1.10 \times 10^{-4}$ & 0.14 \\
\hline & \multirow{2}{*}{158302411} & \multirow{2}{*}{1041586 | F | 0-42 } & Kernel diameter & HD & $7.44 \times 10^{-4}$ & 0.10 \\
\hline & & & Kernel circularity & HD & $4.98 \times 10^{-4}$ & 0.13 \\
\hline \multirow{4}{*}{$6 \mathrm{D}$} & 895318 & 5411945 & Kernel diameter & $\mathrm{H}$ & $1.06 \times 10^{-3}$ & 0.08 \\
\hline & 15112276 & 1099241 | F | 0-17 & Kernel diameter & $\mathrm{H}$ & $2.01 \times 10^{-4}$ & 0.13 \\
\hline & 22055487 & $1019857|\mathrm{~F}| 0-52$ & Kernel diameter & $\mathrm{H}$ & $2.05 \times 10^{-4}$ & 0.10 \\
\hline & 176474631 & 1268158 & Kernel circularity & HD & $5.33 \times 10^{-4}$ & 0.09 \\
\hline 7D & 196638923 & 5331548 & Kernel width & OP & $1.05 \times 10^{-3}$ & 0.08 \\
\hline
\end{tabular}

Under H conditions, 18 significant MTAs were detected (Figure S4, Table 2), i.e., about half of those detected under OP conditions (Table 2). All were located in the A (33\%) and D genomes $(67 \%)$, with no contribution from the B genome (Figure S3c). Chromosome 6A had markers associated with kernel weight, kernel diameter and kernel circularity, whereas chromosome 5D had markers associated with kernel weight and kernel size. Specific markers associated with kernel circularity were located on chromosomes $1 \mathrm{~A}, 4 \mathrm{~A}$ and 3D, and with kernel length on chromosome $4 \mathrm{D}$ (Figure S4, Table 2). $R^{2}$ ranged from 0.08 in markers $987701,1073897|\mathrm{~F}| 0-27$ and 5411945, associated with kernel diameter, kernel size and kernel circularity on chromosome $4 \mathrm{~A}, 5 \mathrm{D}$, and $6 \mathrm{D}$ respectively, to 0.13 in marker 1099241 | F | 0-17, associated with kernel diameter, on chromosome 6D (Table 2). No markers except 1073897 | F | 0-27, associated with kernel size, on chromosome 5D were detected under $\mathrm{OP}$ conditions, indicating their unique association with the kernel traits under $\mathrm{H}$ conditions. Marker 5968258, on chromosome 6A, affected kernel weight and kernel diameter (Figure 2a, Table 2).

Under HD conditions, we detected 43 MTAs (Figure S4, Table 2) with the highest contribution from the D genome (Figure S3d). Chromosome 5D had the highest number of MTAs that were associated with all kernel traits except kernel width; most of them were associated with kernel size (Table 2). We found MTAs associated with kernel size, kernel width and kernel circularity on chromosome 2D and with kernel length on chromosomes 3D and 4D (Figure S4, Table 2). In the B genome, significant MTAs for kernel size were found on chromosome 3B, and for kernel weight, kernel size and kernel circularity on chromosome 5B. In the A genome, MTAs for kernel weight, kernel diameter, kernel size and kernel width were found on chromosome $2 \mathrm{~A}$, for kernel circularity on chromosome $4 \mathrm{~A}$ and for kernel size and kernel perimeter length on chromosome 6A (Figure S4, Table 2). $R^{2}$ ranged from 0.08 for five markers to 0.19 in marker $3940004|\mathrm{~F}| 0-21$, associated with kernel width, on chromosomes 2D (Table 2) and 5A, respectively. Of the 43 MTAs, eight were also identified under $\mathrm{H}$ conditions, indicating their role in plant response to both stresses. On chromosome 2A, marker 1110845 affected kernel weight and kernel diameter, on chromosome 5B, marker 2248796 | F | 0-42 affected kernel weight and kernel size and on chromosome 5D, marker 991465 affected kernel weight, kernel size, kernel length and kernel perimeter length (Figure 2a, Table 2). 


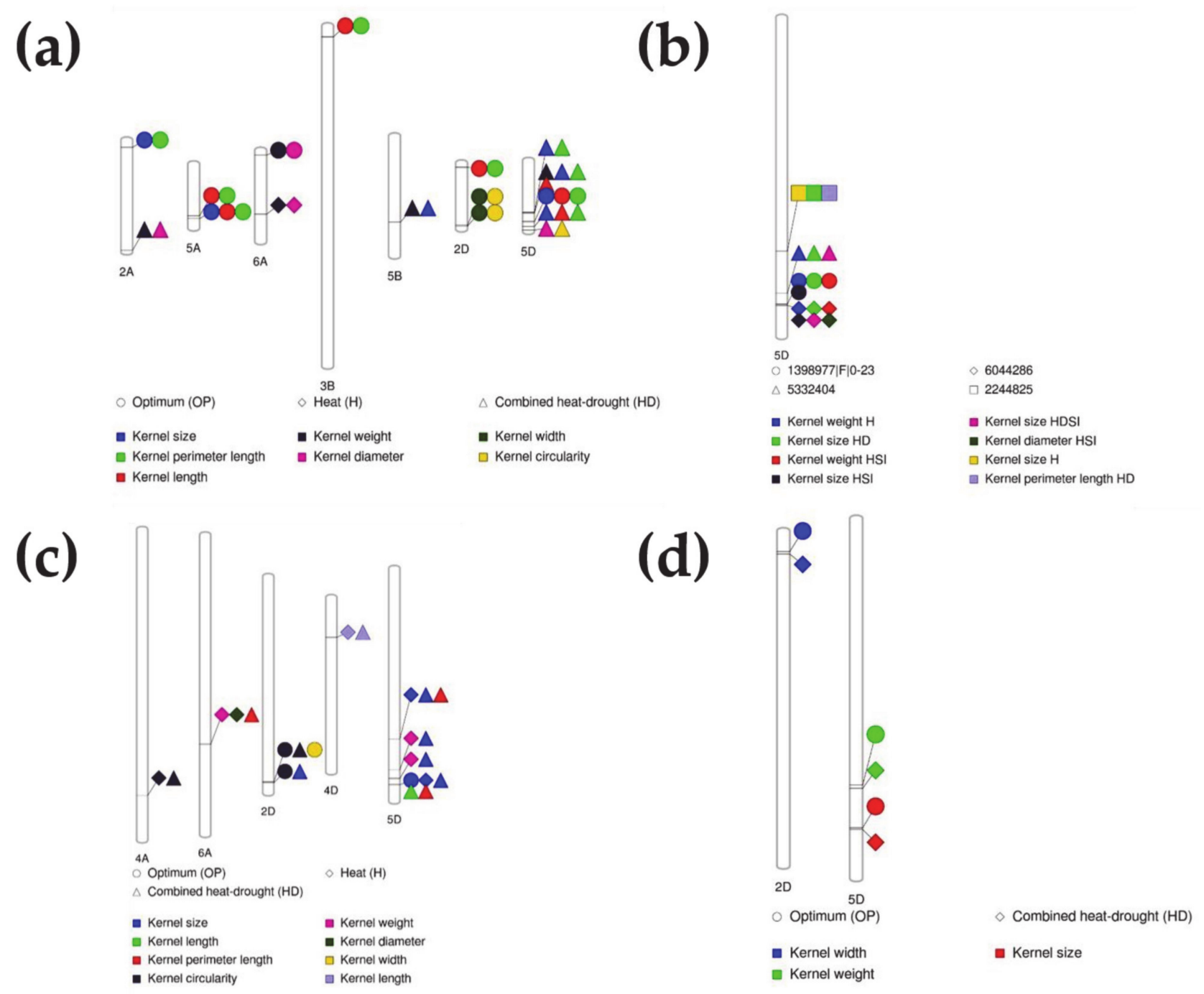

Figure 5. Markers for kernel weight and shape-related traits. (a) Pleiotropic markers under OP, H and HD conditions; (b) markers that showed potential for tolerance for kernel weight under $\mathrm{H}$ conditions and for kernel size under HD conditions and were associated with heat and heat-drought susceptibility indices; (c) stable markers between two or more environments; (d) markers that were located within distances less than $1 \mathrm{cM}$ under different environments.

\subsubsection{MTAs for Heat and Combined Heat-Drought Susceptibility Indices}

On the basis of HSI and HDSI (Table S1), 15 and 29 MTAs, respectively, were identified, of which the D genome contributed 61\% (Table S1). Among them, marker 6044286, on chromosome 5D, was associated with the HSI for kernel weight and kernel diameter, and with both the HSI and HDSI for kernel size (Table S1). We also identified four associations for the HSI for kernel weight and kernel size on chromosome 5D, kernel weight on chromosome 2D and kernel diameter on chromosome 5D (Table S1). $R^{2}$ ranged from 0.08 for marker 1019857 | F | 0-23, associated with the HSI for kernel diameter, to 0.15 for marker 1201315 | F | 0-67, associated with the HDSI for kernel diameter. Of 41 markers, five had pleiotropic effects (Table S1).

We identified four markers (1398977 | F | 0-23, 6044286, 5332404 and 2244825) on chromosome 5D under stress conditions (H and HD) that had pleiotropic effect for kernel weight and kernel size (Figure 5b). Those markers were associated with the HSI and HDSI of kernel weight and kernel size (Figure 5b) — for example, the marker 1398977 | F | 0-23, associated with the kernel size HDSI; 6044286, associated with the kernel size HSI, kernel weight HSI and kernel size HDSI; and 5332404, associated with the kernel size HDSI (Figure 5b). 


\subsubsection{Stable MTAs for Kernel Weight and Shape-Related Traits}

We identified nine stable markers under two or all three conditions, of which seven were on the D genome (Figure 5c). Among them, the marker 1073897 | F | 0-27 was associated with kernel size and stable under $\mathrm{OP}$ and $\mathrm{H}$ conditions and with kernel size, kernel perimeter length and kernel length under HD conditions (Figure 5c). Additionally, markers associated with kernel width on chromosome 2D and with kernel weight and kernel size on chromosome 5D were located close to each other $(<1 \mathrm{cM})$ and were detected under OP and HD conditions (Figure $5 \mathrm{~d}$ ).

\subsubsection{Identification of Putative Candidate Genes for Kernel Weight and Shape-Related Traits}

We searched for the candidate genes associated with the significant markers; we selected the candidate genes that possessed functions associated with kernel shape traits. The putative genes identified are listed in (Table 3). Under the three conditions, OP, $\mathrm{H}$ and HD, we identified genes related to stress tolerance, kernel size and yield regulation in the vicinity of the markers. Among 17 coding proteins in the region of the stable markers 1073897 | F I 0-27 associated with kernel size under OP, H, and HD conditions, we identified a putative RING-type E3 ubiquitin-protein ligase involved in kernel size and yield regulation and encoded by TraesCS5D02G504400 (Table 3). The introduced SNP including $\mathrm{T}$ contributed to increasing the kernel area size under $\mathrm{OP}, \mathrm{H}$ and $\mathrm{HD}$ conditions. This SNP originated from the Ae. tauschii accessions KU-2155 and KU-2156 collected in Iran (Figure 6a-c). The lines MSD187 and MSD128 harboring this allele had higher kernel area sizes than their parent Norin 61 harboring the $C$ allele under all conditions (Figure 6d).
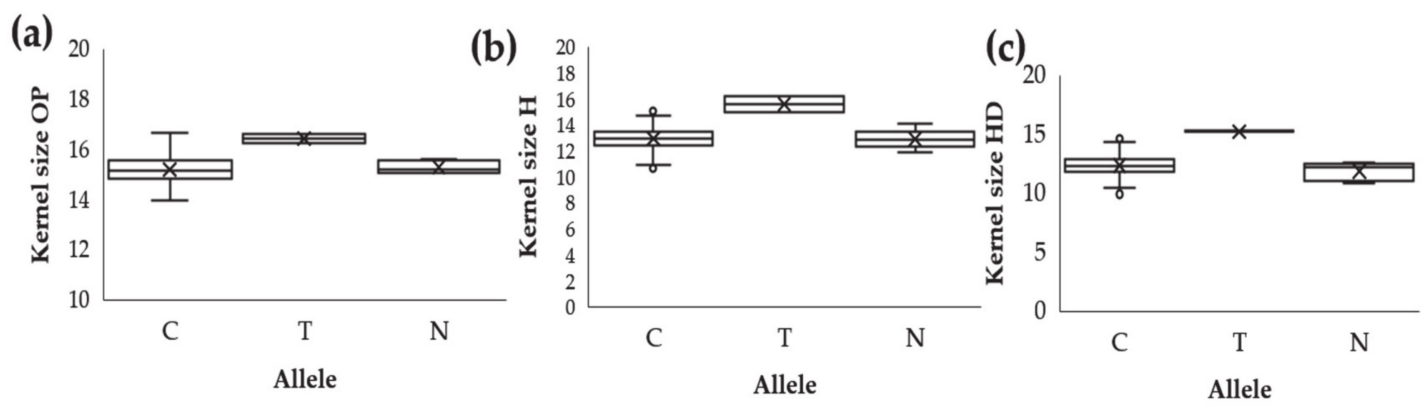

(d)

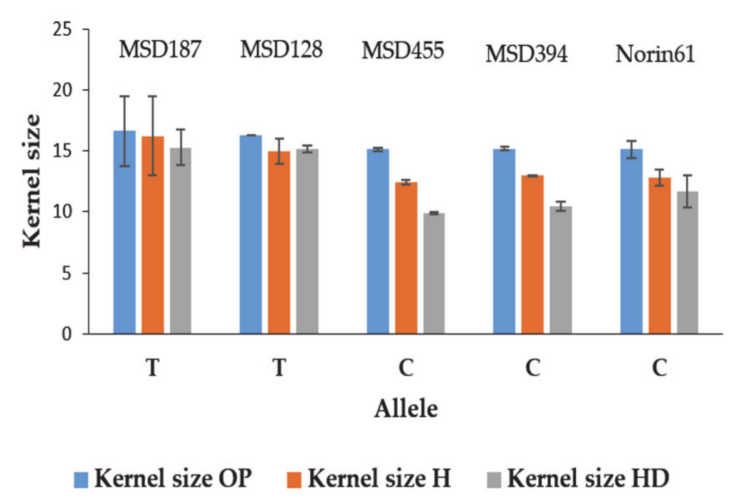

Figure 6. Effects of the alleles of the stable marker SNP_1073897 | F | 0-27 that increases kernel size under (a) optimum, (b) heat and (c) heat-drought conditions. (d) Examples of the lines harboring different alleles for SNP_1073897 | F | 0-27 and their parent Norin 61. 
Table 3. Candidate genes for kernel weight and shape-related traits under optimum, heat and combined heat-drought conditions and their putative physiological roles.

\begin{tabular}{|c|c|c|c|c|c|c|}
\hline Marker & Chromosome & Trait (Environment) & $R^{2}$ & Gene & Protein & Function \\
\hline 5357358 & $2 \mathrm{~A}$ & $\begin{array}{l}\text { Kernel size }(\mathrm{OP}), \\
\text { kernel perimeter } \\
\text { length }(\mathrm{OP})\end{array}$ & 0.11 & TraesCS2A02G099400 & $\begin{array}{l}\text { Basic-leucine } \\
\text { zipper (bZIP) } \\
\text { transcription } \\
\text { factor family } \\
\text { protein }\end{array}$ & $\begin{array}{l}\text { Regulates seed } \\
\text { maturation }\end{array}$ \\
\hline 4407451 & $6 \mathrm{~A}$ & Kernel weight (OP) & 0.10 & TraesCS6A02G056600 & $\begin{array}{l}\text { Auxin- } \\
\text { responsive } \\
\text { protein }\end{array}$ & Regulates auxin \\
\hline 4395641 & $6 \mathrm{~A}$ & $\begin{array}{l}\text { Kernel weight (OP), } \\
\text { kernel diameter (OP) }\end{array}$ & 0.10 & TraesCS6A02G057300 & $\begin{array}{l}\text { F-box domain- } \\
\text { containing } \\
\text { protein }\end{array}$ & $\begin{array}{c}\text { Flower development, } \\
\text { defense response }\end{array}$ \\
\hline 2248796 | F | 0-42 & $5 \mathrm{~B}$ & $\begin{array}{l}\text { Kernel weight (HD), } \\
\text { kernel size (HD) }\end{array}$ & 0.11 & TraesCS5B02G302400 & $\begin{array}{c}\text { Aspartic } \\
\text { proteinase } \\
\text { nepenthesin-1 }\end{array}$ & $\begin{array}{l}\text { Role in drought } \\
\text { avoidance }\end{array}$ \\
\hline 1076033 | F | 0-62 & $2 \mathrm{D}$ & Kernel width (HD) & 0.08 & TraesCS2D02G076500 & $\begin{array}{l}\text { Heat shock } \\
\text { protein Hsp20 } \\
\text { domain- } \\
\text { containing } \\
\text { protein }\end{array}$ & $\begin{array}{c}\text { Tolerance to biotic and } \\
\text { abiotic stresses }\end{array}$ \\
\hline 5332404 & $5 \mathrm{D}$ & $\begin{array}{l}\text { Kernel weight }(\mathrm{H}) \text {, } \\
\text { kernel size }(\mathrm{HD})\end{array}$ & 0.09 & TraesCS5D02G445100 & $\begin{array}{l}\text { Heat stress } \\
\text { transcription } \\
\text { factor }\end{array}$ & $\begin{array}{c}\text { Tolerance to } \\
\text { environmental stress }\end{array}$ \\
\hline 1073897 | F | 0-27 & $5 \mathrm{D}$ & $\begin{array}{l}\text { Kernel size (OP, } \mathrm{H} \text {, } \\
\text { HD), kernel length } \\
\text { (HD), kernel } \\
\text { perimeter length } \\
(\mathrm{OP}, \mathrm{HD})\end{array}$ & 0.08 & TraesCS5D02G504400 & $\begin{array}{l}\text { E3 ubiquitin- } \\
\text { protein } \\
\text { ligase }\end{array}$ & $\begin{array}{l}\text { Regulates grain size } \\
\text { and yield }\end{array}$ \\
\hline 1398977 | F | 0-23 & $5 \mathrm{D}$ & Kernel weight $(\mathrm{H})$ & 0.11 & TraesCS5D02G469900 & $\begin{array}{l}\text { F-box domain- } \\
\text { containing } \\
\text { protein }\end{array}$ & $\begin{array}{l}\text { Flower development, } \\
\text { defense response }\end{array}$ \\
\hline
\end{tabular}

The marker 5332404 on chromosome 5D was associated with kernel weight under $\mathrm{H}$ conditions, kernel size under HD conditions and the kernel size HDSI (Table 2 and Table S1). There were nine coding proteins adjacent to this marker, among them the gene TraesCS5D02G445100 which encodes for a heat stress transcription factor associated with high-temperature stress tolerance (Table 3). The allele A of this marker originated from eight Ae. tauschii accessions (IG 126387, KU-2039, KU-2124, AT 80, KU-20-10, KU-2155, KU-2156 and PI 499262) and increased kernel weight and kernel size under H and HD but not under OP conditions (Figure 7e-g), indicating a special role for this allele under stress. Under stress, the MSD lines harboring the A allele had higher kernel weights and kernel sizes than Norin 61 or MSD lines with the C allele (Figure $7 \mathrm{~d}, \mathrm{~h}$ ). 

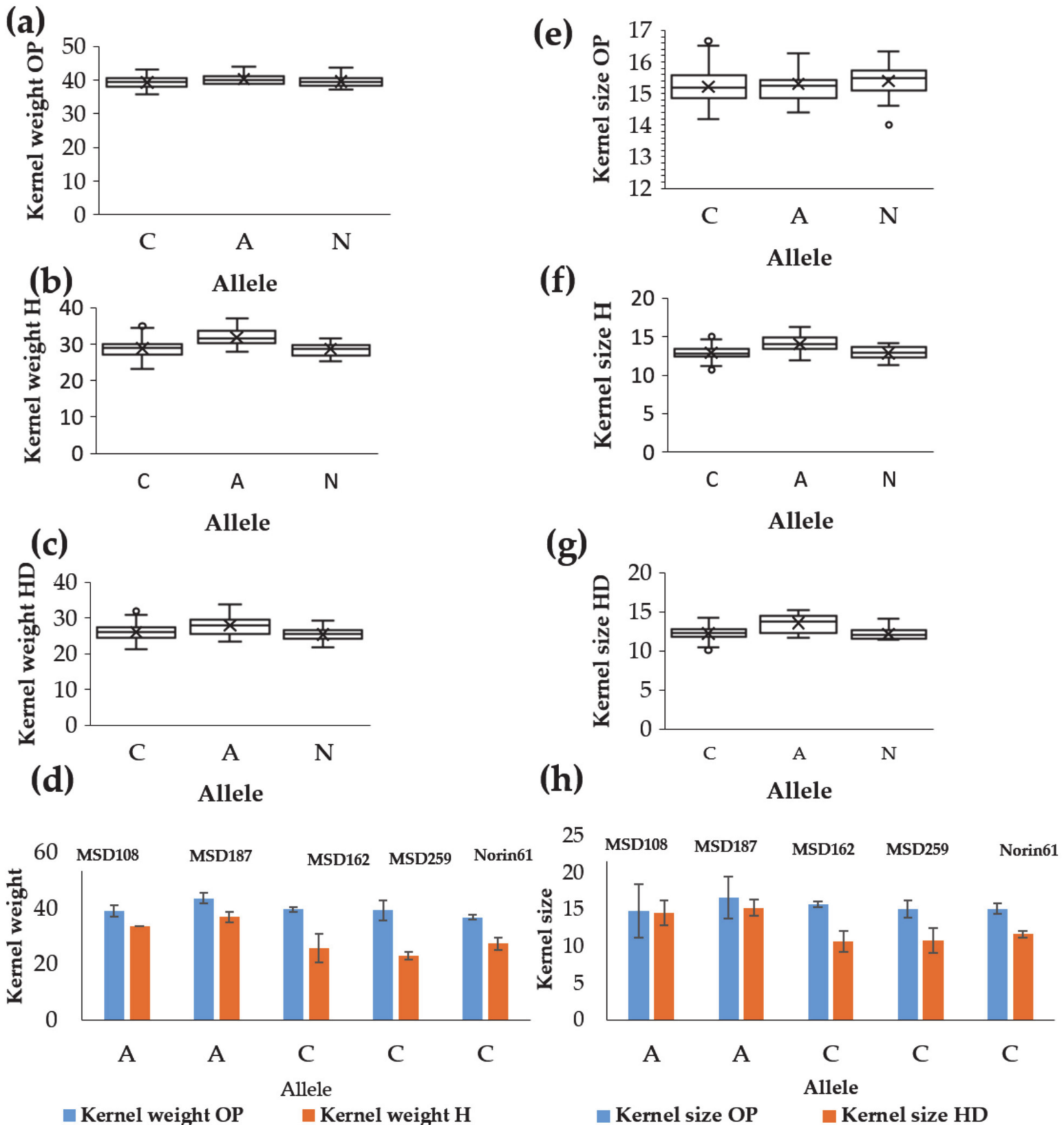

Figure 7. Effects of the alleles of marker 5332404, associated with the kernel weight under heat conditions (H), kernel size under combined heat-drought (HD) conditions and kernel size HD susceptibility index. (a-c) Effects on kernel weight under optimum (OP), H and HD conditions; (e-g) Effects on kernel size under OP, H and HD conditions. (d,h) Effects on kernel weight and kernel size of some MSD lines and Norin 61.

\section{Discussion}

Seed shape and size are the most important agronomic traits owing to their effect on grain kernel weight. Few QTLs associated with kernel traits have been identified under stress conditions in wheat through the association mapping approach. Here, we aimed to detect the effects of heat and heat-drought on kernel weight and shape-related traits using a panel of unique MSD lines harboring different $\mathrm{D}$ genome sources. 


\subsection{Phenotypic Variation for Kernel Weight and Shape-Related Traits under Optimum and Stress Conditions}

The responses to stress conditions were varied for the kernel traits, in which HD conditions severely affecting weight and shape-related traits, followed by the $\mathrm{H}$ conditions. We observed that kernel weight, kernel diameter and kernel size were the traits most affected by HD compared to $\mathrm{H}$ conditions. In addition, Norin 61, the backcross parent of the MSD lines, and the standard check cultivar Imam showed remarkable reductions in kernel weight and shape-related traits under HD compared to H conditions. Combined heat-drought severely affects plants due to heat stress evapotranspiration leading to severe drought stress [26]. Ramya et al. [27] reported that drought and heat stress shorten the grain growth period and lead to improper grain filling, thereby reducing the kernel weight and the overall yield. Moreover, high temperature reduces the conversion of sucrose to starch due to the suppression of the soluble starch synthase enzyme, leading to shriveled kernels [28]. Drought and heat stress accelerate leaf senescence, decrease photosystem II efficiency. As a result, this leads to the reduction of the amount of stored assimilates translocated into developing grains and reduced kernel size $[29,30]$. Heat and drought affect plant growth and thereby reduce yield [31]. In this study, kernel weight was severely affected by HD compared to H conditions. Qasem et al. [32] also observed that combined heat-drought significantly affect kernel weight and yield. Furthermore, shape-related traits, especially kernel diameter and size, were affected by HD compared to $\mathrm{H}$ conditions. However, kernel length was less affected by the stresses than kernel weight and other related traits (Table 1). This finding is in agreement with a report that kernel length is less affected by heat than the kernel weight [33]. We identified some MSD lines, such as MSD187, that could maintain good kernel weight under stress, unlike Norin 61 and Imam. These germplasm lines could be used in wheat breeding programs for heat stress tolerance [34].

Most of the kernel weight and shape traits, including kernel diameter and kernel weight, exhibited moderate heritability under OP conditions, whereas high heritability under $\mathrm{H}$ and $\mathrm{HD}$ conditions was found (Table 1). Xin et al. [35] found high heritability of kernel shape traits in four different environments. Traits with high heritability and genetic advances can be selected directly for crop improvement [36].

The association between kernel weight, kernel diameter, kernel size, kernel width and kernel length under all conditions indicated that all these traits contribute to kernel weight, as reported in $[21,37]$ (Figure 2). Kernel diameter was most strongly correlated with kernel weight, with heritability ranging from 0.64 under OP conditions to 0.88 under stress. These results suggest kernel diameter as a target trait for selection in breeding programs aiming at increasing kernel weight and yield in wheat.

\subsection{Marker Trait Associations for Kernel Weight and Shape-Related Traits under Optimum and Stress Conditions}

More than $50 \%$ of the MTAs identified in this study were on the D genome, thus indicating its higher contribution, especially under stress conditions. This result is inconsistent with previous reports for the kernel shape that indicated a lower contribution of the D genome compared to A and B genomes [20,38,39]. Rasheed et al. [17] also reported a lower contribution of the D genome, though they used synthetic hexaploid wheat. This inconsistency could be attributed to the previous studies being conducted under non-stress conditions and/or to the diversity of the $\mathrm{D}$ genome in the materials used being narrower than the A and B genomes; our results are in agreement with Ali et al. [40]. On the other hand, our findings reveal the uniqueness of the MSD panel as an effective and powerful platform for allele and gene mining in Ae. tauschii.

Thousand-kernel weight is one of the yield components and QTL studies have been conducted for this trait [4]. Also, studies have identified QTLs for grain size at different chromosomes. However, further studies are necessary to understand these traits under stressed environments [41]. Here, in a genome-wide association study (GWAS) for kernel size, we identified a high peak on the chromosome 5D under the HD environment. 
Similar results were described by Afzal et al. [42], specifically, that chromosome 5D influences drought tolerance, indicating that this locus could have an important role in enhancing kernel size and yield under HD conditions. We identified markers associated with kernel weight on chromosomes $6 \mathrm{~A}$ and $5 \mathrm{~B}$ under $\mathrm{H}$ and $\mathrm{HD}$ conditions, respectively. Lopez et al. [41] reported similar results under $\mathrm{H}$ and HD conditions for kernel weight. Under all conditions, the identified MTAs indicated that kernel diameter was most strongly associated with kernel weight. Several studies have reported the association of kernel diameter with kernel weight and other traits under stress condition [43]. These findings further support the above phenotypic correlation findings that kernel diameter, besides other traits, can be a target for selection in breeding programs to increase kernel weight and final grain yield.

Some markers had pleiotropic effects. We found a putative gene, TraesCS6A02G057300, for one of these markers, 4395641, on chromosomes 6A, associated with kernel weight and kernel diameter. This gene encodes an F-box domain-containing protein (Table 3). F-box proteins regulate leaf senescence, flower development and defense responses [44,45]. They also have a role in ethylene signaling [46]. Reduction in ethylene signaling has been suggested to increase grain yield in maize and Arabidopsis [47], consistent with the critical role of kernel weight and kernel diameter in increasing wheat grain yield.

The candidate gene for the pleiotropic marker 2248796 | F | 0-42 for kernel weight and kernel size detected under HD conditions on chromosome 5B encodes aspartic proteinase nepenthesin-1. Yao et al. [48] found that the overexpression of an aspartic proteinase can play a role in drought avoidance through ABA signaling. A recent study in wheat reported that an aspartic proteinase is associated with wheat stress response [49].

Among pleiotropic markers on chromosome 5D detected under $\mathrm{H}$ and $\mathrm{HD}$ conditions, 5332404 had the candidate gene TraesCS5D02G445100. This gene encodes a heat stress transcription factor, which has an important role in responses to abiotic stresses [50]. The lines MSD187 and MSD108, harboring the positive allele of this marker, performed better than Norin 61 and the check cultivar Imam, indicating the usefulness of this marker in maintaining kernel weight under stress. After validation, this marker could be very useful in wheat breeding.

Marker $1076033|\mathrm{~F}| 0-62$, associated with kernel width under HD conditions, on chromosome 2D had the candidate gene TraesCS2D02G076500, which encodes the heat shock protein Hsp20 (Table 3). Heat shock proteins enhance plant immunity [51]. Recently, TaHSP20 genes have been shown to play an important role in abiotic stress tolerance in wheat [52]. Therefore, this marker and its candidate gene could be used in marker-assisted selection programs to improve wheat stress tolerance.

In agreement with Ali et al. [40], we found that kernel area size was most closely associated with kernel perimeter length and then kernel length under all conditions. The candidate gene TraesCS2A02G099400 for marker 5357358 detected under OP conditions on chromosome $2 \mathrm{~A}$ encodes a basic leucine zipper transcription factor (Table 3). Basic leucine zipper is a member of the transcription factor families that controls transcription of seed maturation genes and is expressed during seed development [53].

We identified markers stable under at least two conditions. Marker 1073897 | F | 0-27 was associated with kernel size under all three conditions and is a good candidate for marker-assisted selection in breeding programs. Interestingly, the candidate gene for this marker, TraesCS5D02G504400, encodes a RING-type E3 ubiquitin-protein ligase, like TaGW2 which increases grain size and yield [25]. The ubiquitin pathway plays a crucial role in determining plant seed size [54]. Wheat has TaGW2 copies on chromosomes 6A, $6 \mathrm{~B}$ and $6 \mathrm{D}$. Here, we identified a stable marker and candidate gene under $\mathrm{OP}, \mathrm{H}$ and $\mathrm{HD}$ conditions on chromosome 5D. This study used a panel of MSD derivatives with high diversity of the $\mathrm{D}$ genome derived from several Ae. tauschii accessions; we speculate that the marker identified in our study could be related to a new gene affecting seed size in wheat. However, a detailed study is necessary to confirm this assumption. 


\section{Materials and Methods}

\subsection{Plant Materials}

This study used $400 \mathrm{BC}_{1} \mathrm{~F}_{7}$ multiple synthetic derivative lines developed by crossing and backcrossing the bread wheat (T. aestivum) cultivar Norin 61 and 43 synthetic wheat lines [16]. The synthetic wheat lines were developed by crossing 43 different Ae. tauschii accessions with the durum wheat (Triticum turgidum ssp. durum) cultivar Langdon. We evaluated 400 lines under optimum conditions in Japan and 140 selected lines under stress conditions in Sudan. These 140 lines do not require vernalization treatment and are adapted to Sudanese conditions.

\subsection{Field Experiments}

Optimum conditions in Japan: the 400 MSD lines were grown in a field of the Arid Land Research Center ( $35^{\circ} 32^{\prime} \mathrm{N}, 134^{\circ} 13^{\prime} \mathrm{E}, 11 \mathrm{~m}$ asl), Tottori, Japan, in two seasons: 2015/16 and 2018/19. The soil was sandy (95\% sand, $1.3 \%$ silt, 3.7\% clay) [55]. Before sowing, three commercial fertilizer mixtures-Kumiai Fukugo PKN 366 (MC Ferticom Co., Ltd. Tokyo. Japan; 60 kg), Hitachi Fukugo 1 (Hitachi-kakou Co. Hitachi, Ibaraki, Japan, Ltd.; $40 \mathrm{~kg}$ ) and granular carbonated magnesium lime (Shimizu Kogyo Co., Ltd. Tokyo, Japan; $100 \mathrm{~kg}$ ) —were spread onto the soil. During the tillering stage, Kodo Kasei 444 (Mitsubishi Syoji Agri-service Co., Ltd. Osaka, Japan; 50,000 kg ha ${ }^{-1}$ ) was spread. The experiment was arranged in an augmented randomized complete block design with eight blocks. We used four replicated checks with Norin 61 (the MSD parent), Imam and Tagana (Sudanese heat-tolerant cultivars) and Safedak Ishkashim (a Tajikistan landrace) in each block. Each line was grown in a row of five plants with $0.2 \mathrm{~m}$ between plants. The seeds were sown in late October and plants were harvested in mid-June. The average temperature was $11.9^{\circ} \mathrm{C}$ in the $2015 / 16$ and $11.5^{\circ} \mathrm{C}$ in the $2018 / 19$ seasons. The minimum/maximum temperatures were $-3.8 / 26.2^{\circ} \mathrm{C}$ in $2015 / 16$ and $1.8 / 25.3^{\circ} \mathrm{C}$ in $2018 / 19$; the average temperatures during maturity (May-June) were $20.1^{\circ} \mathrm{C}$ and $19.7^{\circ} \mathrm{C}$, respectively.

Heat $(\mathrm{H})$ and heat-drought (HD) conditions: the lines were grown at the Gezira Research Farm (GRF), Agricultural Research Corporation (ARC), Wad Medani, Sudan $\left(14^{\circ} 24^{\prime} \mathrm{N}, 29^{\circ} 33^{\prime} \mathrm{E}, 407 \mathrm{~m}\right.$ asl), in the 2017/18 season from November to March. We selected GRF because it is recognized as the global center for heat-tolerance research [56,57]. The ARC manages it in collaboration with CIMMYT, ICARDA and Tottori University, Japan (SATREPS Project). This farm is within a clay plain and the soil is heavy clay Vertisol ( $\mathrm{pH}$ 8.5). Before sowing, $\mathrm{P}$ was applied at $18.8 \mathrm{~kg} \mathrm{ha}^{-1}$. Seeds were treated with Gaucho insecticide (imidacloprid, 35\% WP, Bayer Crop Science) at $1 \mathrm{~g} \mathrm{~kg}^{-1}$ seeds and sown at $120 \mathrm{~kg} \mathrm{ha}^{-1}$ manually in the fourth week of November in an alpha lattice design, with two replications. Plot size was four $1.0-\mathrm{m}$ rows with $0.2 \mathrm{~m}$ between rows. In $\mathrm{H}$ plots, plants were irrigated every 10-12 days, as recommended by the ARC. In HD plots, water supply was withheld when $50 \%$ of the lines reached anthesis. Data loggers (Em50, Decagon Devices, Pullman, WA, USA) connected to sensors (Terso21, Decagon Devices) were used to measure soil water potential at $20 \mathrm{~cm}$ depth and the plants were re-watered when the potential reached $-900 \mathrm{kPa}$ to avoid permanent wilting stress. Nitrogen was applied twice as urea at the three-leaf stage (second irrigation) and at the tillering stage (fourth irrigation) at $86 \mathrm{~kg} \mathrm{ha}^{-1}$.

\subsection{Measurement of Kernel Weight and Shape-Related Traits}

From each MSD line in each plot and each replication in all environments, 100 grains were used for kernel trait measurements. Kernel weight and kernel diameter were measured using a single-kernel characterization system (SKCS 4100, Perten Instruments) at the National Agriculture Research Center for Western Region, Fukuyama, Hiroshima, Japan. Kernel shape parameters (area size, perimeter length, length, width, and circularity) were analyzed in SmartGrain software v. 1.2 [58] with up to 100 intact seeds. Circularity was calculated by an equation, $4 \pi$ (area size) $/\left(\right.$ perimeter length) ${ }^{2}$. 


\subsection{Statistical Analysis of Kernel Weight and Shape-Related Traits}

Analysis of variance (ANOVA) for an augmented randomized complete block design was performed using Plant Breeding Tools software (PBTools, v. 1.4, International Rice Research Institute, Laguna, Philippines http:/ /bbi.irri.org/products (accessed on 9 February 2021)). GenStat 18 (VSN International, Rothamsted Research, Harpenden, Hertfordshire, $\mathrm{UK}$ ) was used to carry out the ANOVA for alpha lattice design experiments (H and HD conditions). Broad-sense heritability $\left(H^{2}\right)$ was calculated using PBTools. Pearson's correlation coefficients were performed using R software with a custom script in the ggcorplot package (R core team 2021), available at http:/ / www.sthda.com/english/wiki/ggcorrplotvisualization-of-a-correlation-matrix-using-ggplot2 (accessed on 9 February 2021). Heat susceptibility indices under H (HSI) and HD (HDSI) conditions were calculated for kernel weight, kernel size and kernel diameter as:

$$
\text { HSI }(\text { or HDSI })=(1-Y h / Y) /(1-X h / X),
$$

where $Y h$ is the phenotypic mean of each genotype under $\mathrm{H}$ or HD conditions; $Y$ is the phenotypic mean of each genotype under OP conditions; $X h$ is the mean of all lines under $\mathrm{H}$ or HD conditions; and $\mathrm{X}$ is the mean of all lines under OP conditions.

\subsection{Genome-Wide Association Study and Candidate Gene Identification}

The MSD lines and Norin 61 were genotyped using the DArT-seq platform (Diversity Arrays Technology, Bruce, Australia https://www.diversityarrays.com (accessed on 9 February 2021)) [16]. GWAS for kernel weight and shape-related traits was performed with 14,355 DArT-seq markers in TASSEL5 v. 20,151,113 software [59]. We used a mixed linear model (MLM) with PCA and a kinship matrix to account for population structure and cryptic relationships. Manhattan plots were generated using $-\log _{10}(P)$. The adjusted $P<3 \times 10^{-3}$ was used as a threshold to determine significant association. To identify the candidate genes, significant markers sequences were used for the search in Unité de Recherche Génomique Info, Versailles, France (URGI: https: / / urgi.versailles.inra.fr/ (accessed on 9 February 2021)), with the blast option used for comparisons with the International Wheat Genome Sequencing Consortium, Castanet Tolosan Cedent, France (WGSC) RefSeq V.1 chromosomes. We searched for the candidate genes $0.5 \mathrm{Mb}$ upstream and downstream of the positions of the significant markers.

\section{Conclusions}

In this study, we examined an MSD population with broad diversity in the D genome of bread wheat. The MSD lines were remarkably variable in the kernel traits under OP, $\mathrm{H}$ and $\mathrm{HD}$ conditions. We identified many MTAs, most of which were on the D genome, revealing the power of the MSD lines as a platform for gene mining in Ae. tauschii. Some MSD lines performed better than the backcross parent Norin 61 and the check cultivar Imam under $\mathrm{H}$ and $\mathrm{HD}$ stress conditions. These lines, along with the stable markers, favorable alleles and candidate genes elucidated here, represent a good resource to enhance wheat grain yield under stress and optimum conditions. However, more work will be necessary to validate the suitability of these markers and/or alleles. Nevertheless, our study supports the claim that Ae. tauschii is an important gene reservoir to breed stress-resilient bread wheat.

Supplementary Materials: Supplementary Materials can be found at https:/ / www.mdpi.com/1422 $-0067 / 22 / 4 / 1830 /$ s1. Figure S1. Manhattan plots for kernel weight and shape-related traits under optimum (OP), heat $(\mathrm{H})$ and combined heat-drought (HD) conditions. (a) Manhattan plot for kernel weight; (b) Manhattan plot for kernel size; (c) Manhattan plot for kernel diameter; (d) Manhattan plot for kernel perimeter length; (e) Manhattan plot for kernel length; (f) Manhattan plot for kernel width; (g) Manhattan plot for kernel circularity. Figure S2. Manhattan plot for heat susceptibility index (HSI) and heat drought susceptibility index (HDSI) for (a) kernel weight; (b) kernel diameter; and (c) kernel size. Figure S3. Percent contribution of marker trait associations (MTAs) in the A, B and D genomes 
of bread wheat. (a) All MTAs identified; (b) MTAs identified under OP conditions; (c) MTAs under H conditions; and (d) MTAs under HD conditions. Figure S4. Significant marker trait associations of kernel weight and shape traits under optimum, heat and combined heat-drought conditions. Table S1. Markere trait associations of heat suscibtability index and combined heat drought suscibtability index of kernel weight and shape-related traits in multiple synthetic derivatives lines grown under optimum, heat and combined heat drought conditions.

Author Contributions: H.T. (Hisashi Tsujimoto), H.T. (Hiroyuki Tanaka), N.M.K., Y.S.A.G. and Y.Y. proposed the research idea; G.M.I.E. and K.T. performed the Japan experiment; I.S.A.T. and M.O.I. designed and managed the Sudan experiment; G.M.I.E. and N.M.K. performed statistical analysis; G.M.I.E., Y.S.A.G. and Y.Y. performed the genotypic analysis; G.M.I.E. wrote the manuscript with input from N.M.K.; H.T. (Hisashi Tsujimoto), Y.S.A.G. and N.M.K. critically reviewed the manuscript; Y.Y., I.S.A.T. and K.T. revised the manuscript; H.T. (Hisashi Tsujimoto) and H.T. (Hiroyuki Tanaka) supervised the research. All authors have read and agreed to the published version of the manuscript.

Funding: This study was partly funded by the Science and Technology Research Partnership for Sustainable Development (SATREPS, JPMJSA1805,) by JST, Kakenhi (18H02179), and the Marginal Region Agriculture Project of Tottori University.

Institutional Review Board Statement: Not applicable.

Informed Consent Statement: Not applicable.

Data Availability Statement: Not applicable.

Acknowledgments: The authors are thankful for I. Higashida and K. Inagaki for field assistance in Japan, ARC technical staff for field assistance in Sudan, National Agriculture and Food Research Organization staffs for their kind support during SKCS measurement, and Sachiko Matsunaga for assistance in SKCS measurement.

Conflicts of Interest: The authors declare no conflict of interest.

$\begin{array}{ll}\text { Abbreviations } \\ \text { ANOVA } & \text { Analysis of variance } \\ \text { BLUP } & \text { Best linear unbiased prediction } \\ \text { GWAS } & \text { Genome-wide association study } \\ \text { H } & \text { Heat } \\ \text { HD } & \text { heat-drought } \\ \text { HDSI } & \text { Heat-drought susceptibility index } \\ \text { HSI } & \text { Heat susceptibility index } \\ \text { MSD } & \text { Multiple synthetic derivatives } \\ \text { MTA } & \text { Marker trait association } \\ \text { OP } & \text { Optimum }\end{array}$

\section{References}

1. Weldearegay, D.F.; Yan, F.; Jiang, D.; Liu, F. Independent and Combined Effects of Soil Warming and Drought Stress during Anthesis on Seed Set and Grain Yield in Two Spring Wheat Varieties. J. Agron. Crop. Sci. 2012, 198, 245-253. [CrossRef]

2. Prasad, P.V.; Bheemanahalli, R.; Jagadish, S.K. Field crops and the fear of heat stress-Opportunities, challenges and future directions. Field Crop. Res. 2017, 200, 114-121. [CrossRef]

3. Rad, S.V.; Valadabadi, S.A.R.; Pouryousef, M.; Saifzadeh, S.; Zakrin, H.R.; Mastinu, A. Quantitative and Qualitative Evaluation of Sorghum bicolor L. under Intercropping with Legumes and Different Weed Control Methods. Horticulturae 2020, 6, 78. [CrossRef]

4. Gupta, A.K.; Rather, M.A.; Jha, A.K.; Shashank, A.; Singhal, S.; Sharma, M.; Pathak, U.; Sharma, D.; Mastinu, A. Artocarpus Lakoocha Roxb. and Artocarpus heterophyllus Lam. Flowers: New Sources of Bioactive Compounds. Plants 2020, 9, 1329. [CrossRef] [PubMed]

5. Szliszka, E.; Czuba, Z.P.; Domino, M.; Mazur, B.; Zydowicz, G.; Krol, W. Ethanolic Extract of Propolis (EEP) Enhances the Apoptosis- Inducing Potential of TRAIL in Cancer Cells. Molecules 2009, 14, 738-754. [CrossRef]

6. Yousefi, A.R.; Rashidi, S.; Moradi, P.; Mastinu, A. Germination and seedling growth responses of Zygophyllum fabago, Salsola kali L. and Atriplex canescens to PEG-induced drought stress. Environments 2020, 7, 107. [CrossRef]

7. Schmidt, J.; Claussen, J.; Wörlein, N.; Eggert, A.; Fleury, D.; Garnett, T.; Gerth, S. Drought and heat stress tolerance screening in wheat using computed tomography. Plant Methods 2020, 16, 1-12. [CrossRef] 
8. Su, Z.; Jin, S.; Lu, Y.; Zhang, G.; Chao, S.; Bai, G. Single nucleotide polymorphism tightly linked to a major QTL on chromosome 7A for both kernel length and kernel weight in wheat. Mol. Breed. 2016, 36, 15. [CrossRef]

9. Dholakia, B.B.; Ammiraju, J.S.S.; Singh, H.; Lagu, M.D.; Röder, M.S.; Rao, V.S.; Dhaliwal, H.S.; Ranjekar, P.K.; Gupta, V.S.; Weber, W.E. Molecular marker analysis of kernel size and shape in bread wheat. Plant Breed. 2003, 122, 392-395. [CrossRef]

10. Würschum, T.; Leiser, W.L.; Langer, S.M.; Tucker, M.R.; Longin, C.F.H. Phenotypic and genetic analysis of spike and kernel characteristics in wheat reveals long-term genetic trends of grain yield components. Theor. Appl. Genet. 2018, 131, 2071-2084. [CrossRef] [PubMed]

11. Reynolds, M.; Foulkes, J.; Furbank, R.; Griffiths, S.; King, J.; Murchie, E.; Parry, M.; Slafer, G. Achieving yield gains in wheat. Plant Cell Environ. 2012, 35, 1799-1823. [CrossRef]

12. Tilman, D.; Balzer, C.; Hill, J.; Befort, B.L. Global food demand and the sustainable intensification of agriculture. Proc. Natl. Acad. Sci. USA 2011, 108, 20260-20264. [CrossRef]

13. Zhang, P.; Dundas, I.S.; McIntosh, R.A.; Xu, S.S.; Park, R.F.; Gill, B.S.; Friebe, B. Wheat-Aegilops introgressions. In Alien Introgression In Wheat; Springer International Publishing: New York, NY, USA, 2015; pp. 221-243.

14. Kishii, M. An Update of Recent Use of Aegilops Species in Wheat Breeding. Front. Plant Sci. 2019, 10, 585. [CrossRef] [PubMed]

15. Ogbonnaya, F.C.; Abdalla, O.; Mujeeb-Kazi, A.; Kazi, A.G.; Xu, S.S.; Gosman, N.; Lagudah, E.S.; Bonnett, D.; Sorrells, M.E.; Tsujimoto, H. Synthetic Hexaploids: Harnessing Species of the Primary Gene Pool for Wheat Improvement. Plant Breed. Rev. 2013, 37, 35-122. [CrossRef]

16. Gorafi, Y.S.A.; Kim, J.-S.; Elbashir, A.A.E.; Tsujimoto, H. A population of wheat multiple synthetic derivatives: An effective platform to explore, harness and utilize genetic diversity of Aegilops tauschii for wheat improvement. Theor. Appl. Genet. 2018, 131, 1615-1626. [CrossRef]

17. Rasheed, A.; Xia, X.; Ogbonnaya, F.; Mahmood, T.; Zhang, Z.; Mujeeb-Kazi, A.; He, Z. Genome-wide association for grain morphology in synthetic hexaploid wheats using digital imaging analysis. BMC Plant Biol. 2014, 14, 128. [CrossRef] [PubMed]

18. Chen, G.; Zhang, H.; Deng, Z.; Wu, R.; Li, D.; Wang, M.; Tian, J. Genome-wide association study for kernel weight-related traits using SNPs in a Chinese winter wheat population. Euphytica 2016, 212, 173-185. [CrossRef]

19. Daba, S.D.; Tyagi, P.; Brown-Guedira, G.; Mohammadi, M. Genome-Wide Association Studies to Identify Loci and Candidate Genes Controlling Kernel Weight and Length in a Historical United States Wheat Population. Front. Plant Sci. 2018, 9, 1045. [CrossRef]

20. Su, Q.; Zhang, X.; Zhang, W.; Zhang, N.; Song, L.; Liu, L.; Xue, X.; Liu, G.; Liu, J.; Meng, D.; et al. QTL Detection for Kernel Size and Weight in Bread Wheat (Triticum aestivum L.) Using a High-Density SNP and SSR-Based Linkage Map. Front. Plant Sci. 2018, 9, 1484. [CrossRef] [PubMed]

21. Desiderio, F.; Zarei, L.; Licciardello, S.; Cheghamirza, K.; Farshadfar, E.; Virzi, N.; Sciacca, F.; Bagnaresi, P.; Battaglia, R.; Guerra, D.; et al. Genomic Regions From an Iranian Landrace Increase Kernel Size in Durum Wheat. Front. Plant Sci. 2019, 10, 448. [CrossRef]

22. Chen, Z.; Cheng, X.; Chai, L.; Wang, Z.; Bian, R.; Li, J.; Zhao, A.; Xin, M.; Guo, W.; Hu, Z.; et al. Dissection of genetic factors underlying grain size and fine mapping of QTgw.cau-7D in common wheat (Triticum aestivum L.). Theor. Appl. Genet. 2019, 133, 149-162. [CrossRef] [PubMed]

23. Chang, C.; Lu, J.; Zhang, H.-P.; Ma, C.-X.; Sun, G. Copy Number Variation of Cytokinin Oxidase Gene Tackx4 Associated with Grain Weight and Chlorophyll Content of Flag Leaf in Common Wheat. PLoS ONE 2015, 10, e0145970. [CrossRef]

24. Song, J.; Jiang, L.; Jameson, P.E. Co-ordinate regulation of cytokinin gene family members during flag leaf and reproductive development in wheat. BMC Plant Biol. 2012, 12, 78. [CrossRef] [PubMed]

25. Bednarek, J.; Boulaflous, A.; Girousse, C.; Ravel, C.; Tassy, C.; Barret, P.; Bouzidi, M.F.; Mouzeyar, S. Down-regulation of the TaGW2 gene by RNA interference results in decreased grain size and weight in wheat. J. Exp. Bot. 2012, 63, 5945-5955. [CrossRef] [PubMed]

26. Lamaoui, M.; Jemo, M.; Datla, R.; Bekkaoui, F. Heat and Drought Stresses in Crops and Approaches for Their Mitigation. Front. Chem. 2018, 6, 26. [CrossRef]

27. Ramya, K.T.; Jain, N.; Gandhi, N.; Arora, A.; Singh, P.K.; Singh, A.M.; Singh, G.P.; Prabhu, K.V. Assessing heat stress tolerance and genetic diversity among exotic and Indian wheat genotypes using simple sequence repeats and agro-physiological traits. Plant Genet. Resour. 2015, 15, 208-220. [CrossRef]

28. Jenner, C. Starch Synthesis in the Kernel of Wheat Under High Temperature Conditions. Funct. Plant Biol. 1994, 21, 791-806. [CrossRef]

29. Tian, B.; Talukder, S.K.; Fu, J.; Fritz, A.K.; Trick, H.N. Expression of a rice soluble starch synthase gene in transgenic wheat improves the grain yield under heat stress conditions. Vitr. Cell. Dev. Biol. Anim. 2018, 54, 216-227. [CrossRef]

30. Telfer, P.; Edwards, J.; Bennett, D.; Ganesalingam, D.; Able, J.; Kuchel, H. A field and controlled environment evaluation of wheat (Triticum aestivum) adaptation to heat stress. Field Crop. Res. 2018, 229, 55-65. [CrossRef]

31. Sattar, A.; Sher, A.; Ijaz, M.; Ul-Allah, S.; Rizwan, M.S.; Hussain, M.; Jabran, K.; Cheema, M.A. Terminal drought and heat stress alter physiological and biochemical attributes in flag leaf of bread wheat. PLoS ONE 2020, 15, e0232974. [CrossRef]

32. Qaseem, M.F.; Qureshi, R.; Shaheen, H.; Shafqat, N. Genome-wide association analyses for yield and yield-related traits in bread wheat (Triticum aestivum L.) under pre-anthesis combined heat and drought stress in field conditions. PLoS ONE 2019, 14, e0213407. [CrossRef] 
33. Zhang, Y.; Lou, H.; Guo, D.; Zhang, R.; Su, M.; Hou, Z.; Zhou, H.; Liang, R.; Xie, C.; You, M.; et al. Identifying changes in the wheat kernel proteome under heat stress using iTRAQ. Crop. J. 2018, 6, 600-610. [CrossRef]

34. Elbashir, A.A.E.; Gorafi, Y.S.A.; Tahir, I.S.A.; Elhashimi, A.M.A.; Abdalla, M.G.A.; Tsujimoto, H. Genetic variation in heat tolerance-related traits in a population of wheat multiple synthetic derivatives. Breed. Sci. 2017, 67, 483-492. [CrossRef]

35. Xin, F.; Zhu, T.; Wei, S.; Han, Y.; Zhao, Y.; Zhang, D.; Ma, L.; Ding, Q. QTL Mapping of Kernel Traits and Validation of a Major QTL for Kernel Length-Width Ratio Using SNP and Bulked Segregant Analysis in Wheat. Sci. Rep. 2020, 10, 1-12. [CrossRef]

36. Shokat, S.; Azhar, M.; Nabi, G.; Iqbal, Q. Heritability for characters related to earliness in tomato estimation of heritability and genetic advance for some characters related to earliness in tomato (Solanum lycopersicum L.). J. Agric. Res. 2015, 53, 351-356.

37. Cheng, R.; Kong, Z.; Zhang, L.; Xie, Q.; Jia, H.; Yu, D.; Huang, Y.; Ma, Z. Mapping QTLs controlling kernel dimensions in a wheat inter-varietal RIL mapping population. Theor. Appl. Genet. 2017, 130, 1405-1414. [CrossRef] [PubMed]

38. Chao, S.; Zhang, W.; Akhunov, E.; Sherman, J.; Ma, Y.; Luo, M.-C.; Dubcovsky, J. Analysis of gene-derived SNP marker polymorphism in US wheat (Triticum aestivum L.) cultivars. Mol. Breed. 2008, 23, 23-33. [CrossRef]

39. Wang, S.; Wong, D.; Forrest, K.; Allen, A.; Chao, S.; Huang, B.E.; Maccaferri, M.; Salvi, S.; Milner, S.G.; Cattivelli, L.; et al. Characterization of polyploid wheat genomic diversity using a high-density 90000 single nucleotide polymorphism array. Plant Biotechnol. J. 2014, 12, 787-796. [CrossRef]

40. Ali, A.; Ullah, Z.; Alam, N.; Naqvi, S.S.; Jamil, M.; Bux, H.; Sher, H. Genetic analysis of wheat grains using digital imaging and their relationship to enhance grain weight. Sci. Agric. 2020, 77. [CrossRef]

41. Lopes, M.S.; Reynolds, M.P.; McIntyre, C.L.; Mathews, K.L.; Kamali, M.R.J.; Mossad, M.; Feltaous, Y.; Tahir, I.S.A.; Chatrath, R.; Ogbonnaya, F.; et al. QTL for yield and associated traits in the Seri/Babax population grown across several environments in Mexico, in the West Asia, North Africa, and South Asia regions. Theor. Appl. Genet. 2012, 126, 971-984. [CrossRef]

42. Afzal, F.; Li, H.; Gul, A.; Subhani, A.; Ali, A.; Mujeeb-Kazi, A.; Ogbonnaya, F.; Trethowan, R.; Xia, X.; He, Z.; et al. Genome-wide analyses reveal footprints of divergent selection and drought adaptive traits in synthetic-derived wheats. G3 Genes Genomes Genet. 2019, 9, 1957-1973. [CrossRef] [PubMed]

43. Mwadzingeni, L.; Shimelis, H.; Rees, D.J.G.; Tsilo, T.J. Genome-wide association analysis of agronomic traits in wheat under drought-stressed and non-stressed conditions. PLoS ONE 2017, 12, e0171692. [CrossRef]

44. Chae, E.; Tan, Q.K.G.; Hill, T.A.; Irish, V.F.; Stallock, J.; Molyneaux, K.; Schaible, K.; Knudson, C.M.; Wylie, C. An Arabidopsis F-box protein acts as a transcriptional co-factor to regulate floral development. Development 2008, 135, 1235-1245. [CrossRef]

45. Kim, H.S.; Delaney, T.P. Arabidopsis SON1 Is an F-Box Protein That Regulates a Novel Induced Defense Response Independent of Both Salicylic Acid and Systemic Acquired Resistance. Plant Cell 2002, 14, 1469-1482. [CrossRef] [PubMed]

46. Binder, B.; Walker, J.M.; Gagne, J.M.; Emborg, T.J.; Hemmann, G.; Bleecker, A.B.; Vierstra, R.D. The Arabidopsis EIN3 Binding F-Box Proteins EBF1 and EBF2 Have Distinct but Overlapping Roles in Ethylene Signaling. Plant Cell 2007, 19, 509-523. [CrossRef]

47. Shi, J.; Habben, J.E.; Archibald, R.L.; Drummond, B.J.; Chamberlin, M.A.; Williams, R.W.; Lafitte, H.R.; Weers, B.P. Overexpression of ARGOS Genes Modifies Plant Sensitivity to Ethylene, Leading to Improved Drought Tolerance in Both Arabidopsis and Maize. Plant Physiol. 2015, 169, 266-282. [CrossRef]

48. Yao, X.; Xiong, W.; Ye, T.; Wu, Y. Overexpression of the aspartic protease ASPG1 gene confers drought avoidance in Arabidopsis. J. Exp. Bot. 2012, 63, 2579-2593. [CrossRef]

49. Gou, J.-Y.; Sun, H.-J.; Wang, C.-Y.; Zhang, G.-L. Genomic analyses of wheat Aspartic proteinase gene family provide novel insights for wheat stress responses. SDRP J. Plant Sci. 2020, 4, 174-185. [CrossRef]

50. Guo, M.; Liu, J.-H.; Ma, X.; Luo, D.-X.; Gong, Z.-H.; Lu, M.-H. The Plant Heat Stress Transcription Factors (HSFs): Structure, Regulation, and Function in Response to Abiotic Stresses. Front. Plant Sci. 2016, 7, 114. [CrossRef]

51. Li, J.; Zhao-Hui, C.; Batoux, M.; Nekrasov, V.; Roux, M.; Chinchilla, D.; Zipfel, C.; Jones, J.D.G. Specific ER quality control components required for biogenesis of the plant innate immune receptor EFR. Proc. Natl. Acad. Sci. USA 2009, 106, 15973-15978. [CrossRef] [PubMed]

52. Muthusamy, S.K.; Dalal, M.; Chinnusamy, V.; Bansal, K.C. Genome-wide identification and analysis of biotic and abiotic stress regulation of small heat shock protein (HSP20) family genes in bread wheat. J. Plant Physiol. 2017, 211, 100-113. [CrossRef] [PubMed]

53. Alonso, R.; Oñate-Sánchez, L.; Weltmeier, F.; Ehlert, A.; Diaz, I.; Dietrich, K.; Vicente-Carbajosa, J.; Dröge-Laser, W. A Pivotal Role of the Basic Leucine Zipper Transcription Factor bZIP53 in the Regulation of Arabidopsis Seed Maturation Gene Expression Based on Heterodimerization and Protein Complex Formation. Plant Cell 2009, 21, 1747-1761. [CrossRef] [PubMed]

54. Li, N.; Li, Y. Ubiquitin-mediated control of seed size in plants. Front. Plant Sci. 2014, 5, 332. [CrossRef]

55. Fujiyama, H.; Nagai, T. Studies on responses of plants grown on sand dune soil to a nutrient solution applied by drip irrigation. I. relation between behavior of nutrients in the soil and nutrient uptake by tomatoes. Soil Sci. Plant Nutr. 1989, 35, 55-61. [CrossRef]

56. Tadesse, W.; Suleiman, S.; Tahir, I.; Sanchez-Garcia, M.; Jighly, A.; Hagras, A.; Thabet, S.; Baum, M.; Sherif, A. Heat-Tolerant QTLs Associated with Grain Yield and Its Components in Spring Bread Wheat under Heat-Stressed Environments of Sudan and Egypt. Crop. Sci. 2019, 59, 199-211. [CrossRef]

57. Iizumi, T.; Ali-Babiker, I.-E.A.; Tsubo, M.; Tahir, I.S.A.; Kurosaki, Y.; Kim, W.; Gorafi, Y.S.A.; Idris, A.A.M.; Tsujimoto, H. Rising temperatures and increasing demand challenge wheat supply in Sudan. Nat. Food 2021, 2, 19-27. [CrossRef] 
58. Tanabata, T.; Shibaya, T.; Hori, K.; Ebana, K.; Yano, M. SmartGrain: High-Throughput Phenotyping Software for Measuring Seed Shape through Image Analysis. Plant Physiol. 2012, 160, 1871-1880. [CrossRef] [PubMed]

59. Bradbury, P.J.; Zhang, Z.; Kroon, D.E.; Casstevens, T.M.; Ramdoss, Y.; Buckler, E.S. TASSEL: Software for association mapping of complex traits in diverse samples. Bioinform. Appl. 2007, 23, 2633-2635. [CrossRef] [PubMed] 